\title{
Cosolvent and Dynamic Effects in Binding Pocket Search by Docking Simulations
}

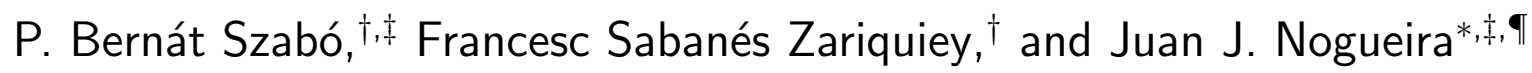 \\ $\dagger$ Department of Chemistry, KU Leuven, 200F 3001 Leuven, Belgium \\ $\ddagger$ Department of Chemistry, Universidad Autónoma de Madrid, Calle Francisco Tomás y \\ Valiente, 7, 28049, Madrid, Spain \\ IIADCHEM, Institute for Advanced Research in Chemistry, Universidad Autónoma de \\ Madrid, Calle Francisco Tomás y Valiente, 7, 28049 Madrid, Spain \\ E-mail: juan.nogueira@uam.es
}

\begin{abstract}
The lack of conformational sampling in virtual screening projects can lead to inefficient results because many of the potential drugs may not be able to bind to the target protein during the static docking simulations. Here, we performed ensemble docking for around 2000 FDA approved drugs with the RNA-dependent RNA polymerase (RdRp) protein of SARS-CoV-2 as target. The representative protein structures were generated by clustering classical molecular dynamics trajectories, which were evolved using three solvent scenarios, namely, pure water and benzene/water and phenol/water mixtures. The introduction of dynamic effects in the theoretical model showed to improve the docking results in terms of the number of strong binders and binding sites in the protein. Some of the discovered pockets were found only for the cosolvent simulations, where the nonpolar probes induced local conformational changes in the protein that lead to the opening of transient pockets. In addition, the selection of the ligands based on a combination of the binding free energy and binding free energy gap between
\end{abstract}


the best two poses for each ligand provided more suitable binders than the selection of ligands based solely on one of the criteria. The application of cosolvent molecular dynamics to enhance the sampling of the configurational space is expected to improve the efficacy of virtual screening campaigns of future drug discovery projects.

\section{Introduction}

Proteins are ubiquitous building blocks playing a critical role in the reproduction, metabolism, and regulation of living organisms and viruses. Understanding and manipulating the way proteins interact with their surrounding is, therefore, of utmost interest from both a biological and a medical point of view. Currently, the most important method to manipulate the function of proteins is through the administering of drugs. For this reason, there exists a growing interest in identifying new binders for a wide variety of proteins in the hopes of treating a number of different sicknesses. ${ }^{1-7}$ In fact, $78 \%$ of the biological drugs approved by the United States Food and Drug Administration (FDA) have clear protein molecular targets. ${ }^{8}$ Therefore, it is not surprising that scientists turned to them once again, when faced with the new and immediate challenges of the coronavirus disease 2019 (COVID-19).

The COVID-19 pandemic caused by the severe acute respiratory syndrome coronavirus 2 (SARS-CoV-2) continues to claim thousands of lives every day more than a year after its outbreak. ${ }^{9}$ However, the knowledge and the developed tools to fight against it are vastly more potent than they were a year before. ${ }^{10}$ Antiviral drugs targeting the proteins vital to the reproduction of SARS-CoV-2 have been the most important tools, ${ }^{11}$ aside from vaccines which are being used as preventative measures. For example, Remdesivir, one of the most widely used antiviral drugs against SARS-CoV-2 around the world, ${ }^{12}$ targets the RNAdependent RNA polymerase (RdRp) protein of the virus. ${ }^{13}$ Furthermore, given the urgency of developing an effective treatment, most attempts to find new inhibitor substances were in fact

drug repurposing studies, targeting the virus's $\operatorname{RdRp}^{14-18}$ or other important proteins. ${ }^{19-22}$ The RdRp protein is an especially promising drug target as it is responsible for the replication 
of the viral RNA inside the host cell, ${ }^{23}$ and it is highly similar to the RdRp of SARS$\mathrm{CoV},{ }^{24}$ which already has a number of verified inhibitors. ${ }^{25}$ In addition, its high-quality three dimensional (3D) structure has been available from as early as April 2020. ${ }^{26}$ In large part, due to the urgent nature of the COVID-19 pandemic, most of the above cited research projects relied heavily, or even exclusively, on computational techniques for the discovery of the potential inhibitors, due to the cost and time efficiency of such methods.

High-throughput screening enables the routinely evaluation of thousands of substances in a week. ${ }^{27}$ This tremendous efficacy is often supported by the development and application of innovative computational methods, which became more useful since the advent of structurebased drug design, where potential drugs are created or found based on the 3D structure of the protein target. ${ }^{28,29}$ Although such target structures were initially only obtainable through costly and cumbersome experimental methods, such as X-ray crystallography ${ }^{30}$ or nuclear magnetic resonance (NMR) spectroscopy, ${ }^{31}$ they are nowadays much more readily available due to the gradual improvement of existing methods, the appearance of new experimental methods, such as cryo-electron microscopy, ${ }^{32}$ and the development of recent computational techniques, such as homology modeling. ${ }^{33}$ Taking advantage of the quickly growing body of available genomic data, computational tools capable of predicting protein structures from mere amino acid sequence information have also been developed. ${ }^{34-36}$ By employing one (or a combination) of the above techniques, high-quality structures are available for a larger number of protein targets than ever before.

The current challenge to computational chemists is therefore how to best utilise the available structural information. The computational methods developed for structure-based drug design fall into two main categories: de novo design methods construct new, tailored ligands, while docking methods select ligands complimentary to the target from the existing compound space. ${ }^{37}$ Although different methodologies have been widely employed to investigate the binding of ligands to proteins, such as Monte Carlo techniques ${ }^{38,39}$ and Gaussian accelerated molecular dynamics (MD), ${ }^{40,41}$ the vast majority of theoretical drug design 
investigations rely on docking calculations due to their computational efficiency. Among the docking methods, virtual screening (VS) has emerged as a particularly successful technique. $^{7,42}$ This procedure can be thought of as a computational extension to high-throughput screening, where a large number of compounds are docked to the target protein structure in silico. Traditionally, VS campaigns have been carried out utilising a single, experimentally determined protein structure, often in the crystallised form. ${ }^{37,43}$ However, the deficiencies of using only a single crystallised protein structure has been recently recognised. ${ }^{37,43-46}$ Firstly, the structure of the crystallised protein often differs significantly from the conformations that the protein adopts in vivo. Secondly, even if the crystal structure is representative of the conformation most often visited in solution, a single structure cannot account for the dynamics of protein motion.

Different theoretical models that consider the importance of protein motion have been developed at the cost of computational efficiency, e.g., the induced-fit model of ligand docking, ${ }^{47,48}$ where the structure of the protein may change during ligand uptake, or the model of conformational selection, ${ }^{49-51}$ which views the target protein as a dynamic object even in the absence of ligands. The need to take protein flexibility and motion into account became even clearer with the discovery of cryptic or hidden pocket structures. ${ }^{52-54}$ The characteristic property of these pockets is that they only appear in the presence of the appropriate ligand, while their existence is not obvious from the equilibrium structure of the protein. The exact mechanism of their formation is not yet clear, although some combination of induced-fit and conformational selection has been hypothesised. ${ }^{53}$ The discovery and theoretical description of such pockets are hindered by the fact that their opening often requires large scale rearrangements of the protein structure, events that are traditionally hard to predict with computational techniques. ${ }^{55}$

With the importance of protein dynamics gaining wider recognition, new and more elaborate methods are appearing which aim to account for this phenomenon. On the one hand, some of the modern computational docking programs, such as AutoDock Vina, ${ }^{56}$ can treat 
a selected number of protein residues as flexible at the cost of increased calculation times. This method is well suited to study a previously known, specific binding site of the protein. However it cannot account for larger structural changes of the protein and is limited to a handful of flexible residues due to its computational requirements. On the other hand, the family of ensemble docking techniques utilises traditional (rigid protein) docking calculations in combination with an ensemble of protein conformations to account for the flexibility of the target. ${ }^{37,43}$ The careful selection of the structures of the ensemble can enable the description of large scale conformational changes and to the discovery of new cryptic pockets. ${ }^{51,54,55}$ The main challenge for these methods is the generation of the protein structure ensemble, which can be achieved experimentally by using different crystallised structures, ${ }^{37,57,58}$ or computationally by, e.g., conformational space searches, ${ }^{59}$ neural networks ${ }^{60}$ and MD. ${ }^{43,61}$

MD is an especially promising avenue, after all it has been designed for the very purpose of efficiently sampling the conformational space of proteins and other large molecules. However, one of the most important obstacles of MD calculations is the extremely slow convergence of the calculated trajectories, ${ }^{43}$ which precludes the population of rarely visited conformations. Even with highly specialised codes and computers the longest timescales reachable are in the range of milliseconds. ${ }^{62}$ In order to be able to sample rare events, a number of modified MD techniques have been developed. The first group of these is the enhanced sampling methods, where some unphysical bias is introduced into the simulation in order to encourage the sampling of otherwise unlikely conformations. Some of the most popular enhancedsampling methods in the context of cryptic pocket discovery are umbrella sampling, ${ }^{63}$ steered $\mathrm{MD},{ }^{64}$ metadynamics, ${ }^{65}$ and replica exchange $\mathrm{MD},{ }^{66}$ among others. A completely separate approach for the sampling of rarely visited conformations harboring cryptic pockets is that of the cosolvent methods. The main idea behind these frameworks is to replace the traditional water solvent in MD simulations with a mixture of water and some other cosolvent. The oftentimes hydrophobic or amphipatic cosolvent probes can then interact with the protein and occasionally induce conformational changes or stabilise some conformations where a 
cryptic pocket is open. Cosolvent methods have been successfully used to identify cryptic sites in a number of targets. ${ }^{51,54,67-72}$

The primary aim of the present work is to investigate the effect of protein dynamics in the results of a VS campaign. The ensemble of protein structures is obtained via MD simulations. Further sampling is achieved by cosolvent trajectories where water/benzene and water/phenol mixtures are employed. Recognising the severity of the COVID-19 pandemic, the calculations are carried out on the RdRp protein of SARS-CoV-2 and a set of FDA approved small molecule drugs, in the hopes of contributing to the generation of knowledge necessary to develop effective treatments against this virus. We show that the introduction of protein dynamics in VS significantly improves the chances to find suitable binders and unveil potential new binding sites in the protein. This is especially relevant considering the significant amount of work published in the last year aimed to discover efficient antiviral agents against the SARS-CoV-2 virus based on static docking calculations that neglect dynamic effects.

\section{Computational Details}

\section{Setup and Molecular Dynamics Simulations}

The SARS-CoV-2 RdRp protein complex was chosen as the target of our investigations. In its active form it is composed of three domains, namely, the nonstructural proteins (NSP) 7, 8 and 12 of SARS-CoV-2. Its active site is located in a deep groove at the boundary of the palm and thumb domains of its NSP12 unit, ${ }^{14}$ and is highly similar to that of the analogous protein of the SARS-CoV. ${ }^{25}$ Its simulation ready structure was obtained from the website of D. E. Shaw Research, ${ }^{73}$ where extensive MD simulations have already been carried out for it. In Reference 14, the authors note that two zinc ions are necessary for the structural integrity of the protein. These ions were however not found in the structures and trajectories downloaded from D. E. Shaw Research. After numerous failed attempts at stabilising these zinc ions in 
their bound positions with restraining potentials and gradual heating, their inclusion was rejected in favor of the original D. E. Shaw structure. Additionally, the crystal structure in its apo form determined with cryo-electron microscopy was also downloaded from the Protein Data Bank website (6M71) ${ }^{74}$ to carry out docking calculations, as explained below.

The MD calculations were carried out with the Amber 18 program package, ${ }^{75}$ according to the following protocol. The protein was described by the ff14SB force field, ${ }^{76}$ water by the TIP3P model, ${ }^{77}$ the cosolvents benzene and phenol by the general Amber force field for organic molecules, ${ }^{78}$ and the sodium ions by suitable Amber force field parameters. ${ }^{79}$ Three types of solvent boxes were prepared for the simulations: a simple water one and two with either benzene or phenol as cosolvent. The cosolvent boxes were built with the help of the packmol program. ${ }^{80}$ The concentration of the cosolvents were set to $10 \mathrm{v} / \mathrm{v} \%$. In the case of benzene, severe clustering of the cosolvent molecules was observed during the MD simulations when the default force field parameters were used. To circumvent this issue, scripts included in the ParmEd distribution ${ }^{81}$ were utilised to introduce Lennard-Jones potentials for the $\mathrm{C}$ atoms of the benzene molecules with parameter values of $\gamma=0.00036$ $\mathrm{kcal} / \mathrm{mol}$ and $R_{\min }=7.12719 \AA$ (the default values used in ParmEd). After this modification was made, no clustering of the benzene molecules was observed during the simulations.

The protein structures were solvated using the tleap program of Amber $18^{75}$ in octahedral solvent boxes, where a distance of at least $12 \AA$ was left between any atom of the protein and all sides of the solvent box. The charge of the system was neutralised with sodium ions. During the simulations, the particle-mesh Ewald method ${ }^{82}$ with a grid spacing of $1.0 \AA$ was used to compute the electrostatic interactions and a $12 \AA$ cutoff for the nonbonded interactions was chosen. The solvated systems were first minimised for 1000 gradient descent steps followed by 1000 conjugate gradient steps. Next, the heating of the systems to $300 \mathrm{~K}$ were performed during a $1 \mathrm{~ns}$ simulation with the Langevin thermostat (collision frequency of 1.0 $\mathrm{ps}^{-1}$ ) and with a 2.0 fs timestep in the NVT ensemble. Finally, production simulations were carried out at $300 \mathrm{~K}$ and 1 bar pressure using the Langevin thermostat (collision frequency 
of $1.0 \mathrm{ps}^{-1}$ ) and Berendsen barostat (relaxation time of $2.0 \mathrm{ps}$ ) in the NPT ensemble, using a timestep of 2.0 fs. Specifically 3 replicas of 200 ns each were run for the production calculation for each solvent. The last 2 simulations for each solvent were started from a random equilibrated frame of the first simulation for that solvent, with the velocities of all particles randomised according to the Boltzmann distribution at $300 \mathrm{~K}$. The production calculations were run with GPU acceleration, using the CUDA version of the pmemd program of Amber $18 .^{83}$

For the clustering of the MD trajectories the cpptraj program ${ }^{84}$ of Amber 18 was utilised. A density based clustering algorithm (chosen with the dbscan keyword of cpptraj) was employed, with the parameters $k$ (unitless) and $\varepsilon$ set to 4 and $1.1 \AA$, respectively, as will be explained below. For each type of solvent the equilibrated part of the first production simulation and the two additional replicas were concatenated and the clustering was carried out separately for each solvent. Before clustering, the structures in every frame were aligned to each other by their alpha carbon atoms. The clustering was performed using the RMSD values of the alpha carbon atoms as the distance metric between the conformations. A total of 19 cluster representatives were obtained with 13 structures coming from the trajectory with water as the solvent, while the benzene and phenol cosolvent trajectories yielded 3 cluster representatives each.

\section{Docking Calculations}

The set of FDA approved drugs were downloaded from the ZINC database ${ }^{85}$ in the mol2 format. This set is a popular choice for drug repurposing studies ${ }^{16-18}$ and, with approximately 2000 contained ligands, it was feasible to perform docking calculations for all protein conformation/ligand pairs. From this set, 1957 ligand structures were converted to the pdb format, necessary for docking with AutoDock Vina, with the openbabel program. ${ }^{86}$ The 19 cluster representative protein structures along with the apo crystal structure were aligned to each other by the RMSD distances between their alpha carbons. The protein and ligand 
structures were prepared for docking, relying on the scripts included in the AutoDockTools 4 distribution. ${ }^{87}$ The same docking region, generated by AutoDockTools 4, was used for all docking calculations, which encompassed the whole protein structure. To carry out the docking calculations, AutoDock Vina was run with the default command line options, except for the exhaustiveness option which was increased to 24, as suggested by the authors for large docking regions, and the num_modes option which was set to 20 to obtain the 20 best poses for each ligand. The parallel execution of the docking calculations were managed with in-house scripts. Attending to the results of the docking calculations the best ligands binding to each discovered pocket were selected based on two different criteria, namely, binding free energy and binding free energy gap between the two best poses of each ligand, which will be discussed below.

\section{Pocket Description}

The binding sites of the protein, discovered through computational docking calculations, were analysed with the mdpocket program, ${ }^{88}$ part of the fpocket distribution. To this end, the 19 cluster representative protein structures were aligned to each other by their alpha carbons and concatenated to create a mock trajectory readable by mdpocket. The regions of space which the discovered binding pockets occupy were selected manually, by inspecting the poses of the ligands binding to the pocket in question. Based on the suggestions of the mdpocket authors, large regions were selected for each pocket, encompassing all or almost all docked ligand poses. With the protein structures concatenated and the binding regions selected, mdpocket was run with the $-\mathrm{S}$ option, instructing the program to score pockets by their druggability. Among its results mdpocket provides a number of pocket descriptors calculated for each frame of the supplied trajectory. From these descriptors, the pocket volume is utilised in the present study to discuss of stability of the pockets. 


\section{Results and Discussion}

\section{Equilibration of the Trajectories}

In this section, first the equilibration of the protein during the various (cosolvent) MD trajectories is examined by plotting the RMSD of the protein structure from its initial state throughout the simulated time. On top of the usual task of selecting the equilibrated part of each trajectory to be considered for further analysis, these plots are useful to detect potential differences in the equilibration process between the water solvent and the cosolvent trajectories. On Figure 1A,C,E such plots obtained for the first replica of $200 \mathrm{~ns}$ of each solvent type are shown. The protein structures are well equilibrated after 50 ns of simulation time in water and in benezene/water solvents. In the case of phenol/water, significant variations are still observed after $100 \mathrm{~ns}$, a fact that could indicate that the cosolvent probes have stabilised some protein conformations that are not often visited with water as the solvent, and that are farther from the original protein conformation than those appearing frequently in water based simulations. This is further investigated by plotting the average residue-wise RMSD values in order to monitor the mobility of the different regions of the protein. This data is shown on Figure 1B,D,F for the first production replicas of all solvents. The initial fraction

of the trajectories where the RMSD suffers a strong increase, which correspond to 40, 20 and $10 \mathrm{~ns}$ for water, benzene/water and phenol/water, respectively, was not considered. To make the plots more readable, the moving average of residue mobility calculated with a symmetric window with a width of nine residues is plotted. The numbering of the residues on this figure simply describes the order in which Amber18 considers them, therefore these numbers cannot be straightforwardly compared with another numbering published in the literature.

At first glance the three plotted curves of Figure 1B,D,F appear fairly similar: they showcase higher mobility of the terminal residues and lower average RMSD values for residues between 200 and 800 . The section between residues 600 and 800 (the interface of the palm and thumb domains of the core NSP12 unit) is roughly where the active site of the protein 

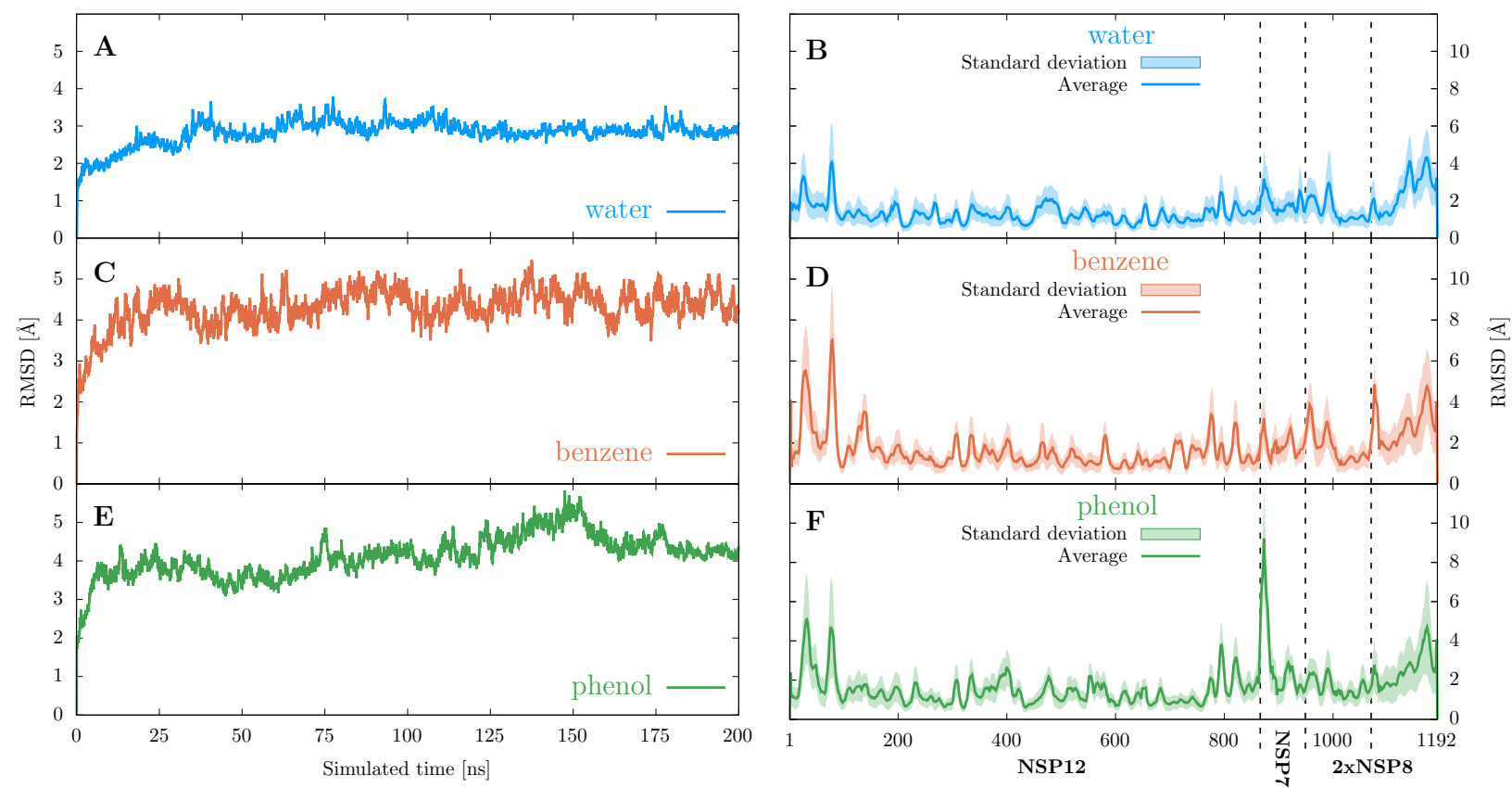

Figure 1: On the left side (panels A, C and E), the evolution of the RMSD distance of the protein from the starting conformation during the MD trajectories. The first replica for each solvent is plotted. On the right (panels $\mathrm{B}, \mathrm{D}$ and $\mathrm{F}$ ), average RMSD values for the protein residues in the three different solvent trajectories. The transparently coloured region around the average values represents the standard deviation throughout the simulation. The first replica for each solvent is plotted. The location of the NSP domains of the protein is indicated by the dashed vertical lines. 
is located. Since the conformation of the residues around the active site is crucial for the activity of the protein, it is not surprising that these regions are more stable in the absence of substrates, than some other less critical areas. Upon more careful inspection, some differences between the three mobility plots can be identified. Most notable are the unusually high RMSD values around residues 870-880 observed in the trajectory with phenol as the cosolvent. These residues correspond to the terminal regions of the NSP12 and NSP7 units of the RdRp complex and, therefore, their higher mobility in itself is not surprising. The fact that the RMSD values are outstandingly high only in the phenol trajectory could indicate some occasional interaction of these residues with the phenol probes, and could explain the higher RMSD observed in Figure 1E after 100 ns of simulation. A further difference between the three curves is the larger variance of the per residue RMSD values observed when the cosolvent MD trajectories are considered. Firstly, this manifest itself in the slightly larger standard deviations shown for these trajectories, especially around residues 1100-1192, where larger standard deviation areas can be observed for the cosolvent trajectories with similar average mobility for all three trajectories. Secondly, the differences between the average RMSD values between neighboring residues are also in general larger for the two cosolvent trajectories. This results in much deeper valleys and higher, more pronounced spikes in Figure 1D,F than in Figure 1B. This latter observation can lead one to assume that the

effects of the cosolvent probes are quite local in nature: they can significantly change the conformation of the handful of residues they are directly interacting with but leave the larger scale structure of the protein more or less intact.

\section{Selection of Representative Protein Conformations}

As mentioned above, the dbscan algorithm of cpptraj is used to perform the clustering of the trajectories. The clustering is carried out separately for the three solvents, with the three replicas of each of them concatenated and treated as a single trajectory, but excluding the first 40, 20 and $10 \mathrm{~ns}$ for water, benzene/water and phenol/water, respectively, where 
the RMSD of the protein is not stabilized. In order to carry out a successful clustering of the trajectories, first the $k$ and $\varepsilon$ parameters of the density based clustering algorithm have to be tuned. On top of performing this tuning of the parameters, the effects of considering only the alpha carbon atoms for the RMSD calculations instead of all heavy atoms of the protein are also evaluated. Finally, possible redundancies in the set of representative protein structures are investigated.

The tuning of the parameters of the dbscan algorithm is performed by systematically varying the values for these parameters to see which combination yields the most optimal clustering. The computation of the RMSD distances between all frames of a trajectory is much more demanding if on top of the alpha carbons, all other heavy atoms are considered as well. To speed up these computations, the technique of sieving is utilised: only every other frame is considered explicitly during the clustering, the remaining frames are simply added to the cluster with the cluster representative most similar to them. To measure the quality of the clustering, four metrics are utilised. Two of these, namely the Davies-Bouldin index (DBI) and the pseudo-F statistic (pSF), aim to compare the intra- and intercluster variances, with a small intracluster and large intercluster variance indicating good quality clustering. ${ }^{89}$ Since both of these scores are heavily influenced by the number of obtained clusters, the comparison of their absolute values between different MD trajectories has limited meaning. Instead, the trends arising in these metrics through the systematic variation of the clustering parameters can be interpreted to optimise these parameters. At this point it is useful to mention that low values of DBI and high values of pSF are desirable. The other two descriptors utilised to describe the quality of the clustering are the number of noise frames (frames not included in any cluster), and the number of clusters defined by the algorithm. The number of noise frames should clearly be kept low to avoid missing any important conformations only because it is visited very rarely and is therefore considered an outlier by the algorithm. Finally, while a high number of clusters is desirable, as it can result in a wider variety of protein conformations, the computational limitations of performing explicit 
docking calculations to each representative conformation with thousands of ligands should be kept in mind.

On Figure 2, the descriptors of the water solvated trajectory clustering can be seen for the case when only the alpha carbons are considered during the RMSD distance calculations. As it can be seen, considering $\varepsilon$ values larger than $1.2 \AA$ leads to a single obtained cluster (Figure 2D), for which the clustering descriptor metrics cannot provide a meaningful value. Since a single cluster is clearly not ideal, these large $\varepsilon$ values do not need to be considered during the search for the optimal parameters. Focusing instead on parameter $k$, the most significant differences between the different values for this parameter can be discovered on the pSF bahaviour (Figure 2B). Here, the curves with $k=4$ or 6 , reaching their peak at $\varepsilon=1.1 \AA$, are clearly superior to the other two. Contrary, the variation of $k$ has much more limited effects on DBI, as shown in Figure 2A. In fact, all DBI curves are more or less constant if $\varepsilon$ is smaller than or equal to $1.1 \AA$, at which point the DBI values drop rapidly and become zero at $1.2 \AA$. Considering the behaviour of the pSF and DBI descriptors, values of $\varepsilon=1.1 \AA$ and $k=4$ are promising candidates to be the optimal choice. Further advantages of this choice can be seen by looking at Figure 2C,D: the number of noise frames stay below $2 \%$, while a reasonable number of clusters (thirteen) is obtained. The thirteen clusters resulting in thirteen representative protein conformations is deemed suitable number both because docking to these conformations represents a manageable computational challenge, and because similar numbers of clusters have been reported in the literature for comparable MD trajectories. ${ }^{1,2}$

As a comparison, similar descriptors are calculated for the clusterings when all heavy atoms are considered during the RMSD calculations. The trends observed in this case look very similar to those discussed when only the alpha carbons are considered, therefore these plots are omitted. The only notable difference is that significantly larger $\varepsilon$ values are needed to obtain similar results as in the case when only the alpha carbons are considered. This phenomena can be explained if higher mobility is assumed for the non-backbone heavy 

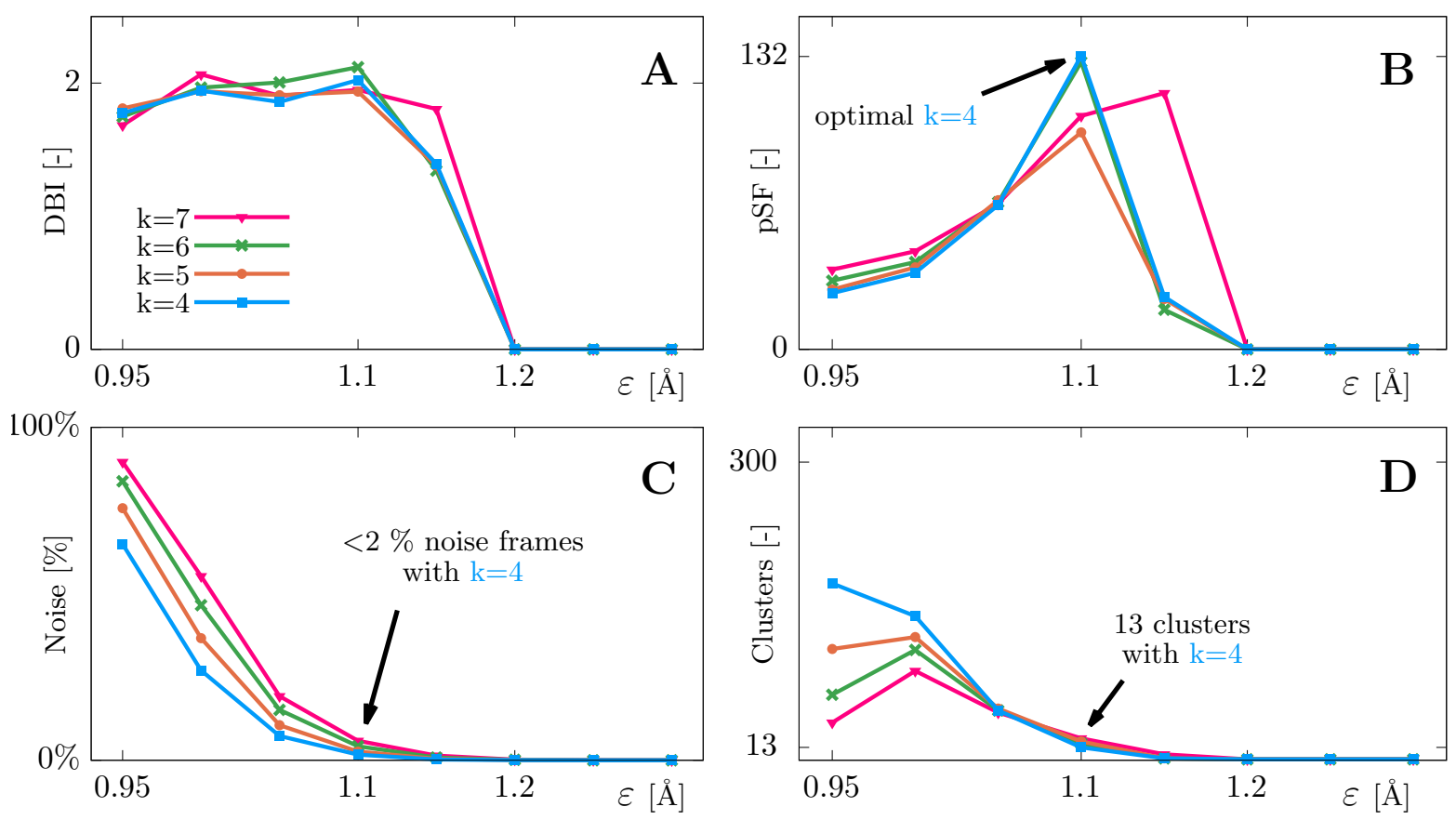

Figure 2: Clustering descriptors utilised for the tuning of the dbscan parameters: (A) the Davies-Bouldin index (DBI), (B) the pseudo-F statistic (psF), (C) the percentage of noise frames, and (D) the number of clusters. The descriptors were obtained by clustering the MD trajectory with water as the solvent and considering only the alpha carbon atoms for the RMSD calculations. The $\varepsilon$ parameter in units of Angströms are shown on the horizontal axes in all cases, while on the vertical axes the various unitless descriptors are shown. 
atoms of the protein in comparison to the alpha carbons. Since no clear advantage of the all heavy atom clustering is found, the significantly increased computational costs of considering much more atoms for the RMSD calculations make this type of clustering an inferior option compared to considering only the alpha carbons.

The performance of the clustering algorithm is also examined on the benzene cosolvent trajectory, with only the alpha carbons considered for the RMSD distance calculations, to investigate any potential differences in the quality of the clustering due to the presence of cosolvent probes during the simulation. The same descriptors as in the case of the water solvated trajectory are plotted on Figure 3. Contrary to the water trajectory, in this case the number of clusters does not decrease to a single one at higher $\varepsilon$ values, but instead is saturated at three, see Figure 3D. As a consequence, the DBI and pSF values do not vanish for these values of $\varepsilon$ (Figure 3A,B). Instead, a sudden shift can be observed between $\varepsilon=1.0 \AA$ and $1.1 \AA$ for both metrics, while for values higher or lower than these, the curves are more or less constant. The facts that this shift is occurring near $\varepsilon=1.1 \AA$, and that this value is already in the more favorable interval for both metric curves (small values of DBI and high values of $\mathrm{pSF}$ ), highlight the attractiveness of choosing $1.1 \AA$ as the value of the $\varepsilon$ parameter. The pSF value at $\varepsilon=1.1 \AA$ of the curve associated with $k=4$ is again one of the best along with $k=5$. Figure 3C,D reveals no surprises: the number of noise frames is negligible with the parameter values being considered, while the number of clusters stagnates around the reasonable value of 3 and increases sharply only for $\varepsilon$ values lower than $1.0 \AA$. Similar plots have been created for the trajectory with phenol as the cosolvent, however it showed very similar characteristics as this one, therefore it is not displayed here. To summarise, the clustering parameters values of $\varepsilon=1.1 \AA$ and $k=4$ prove to be ideal choices, as they result in a clustering that is suitable for our purposes for all types of trajectories considered. The resulting clustering yielded 19 total representative protein conformations (13 from the water, 3 from the benzene/water and 3 from the phenol/water trajectories), which were utilized during the subsequent ensemble docking calculations. 

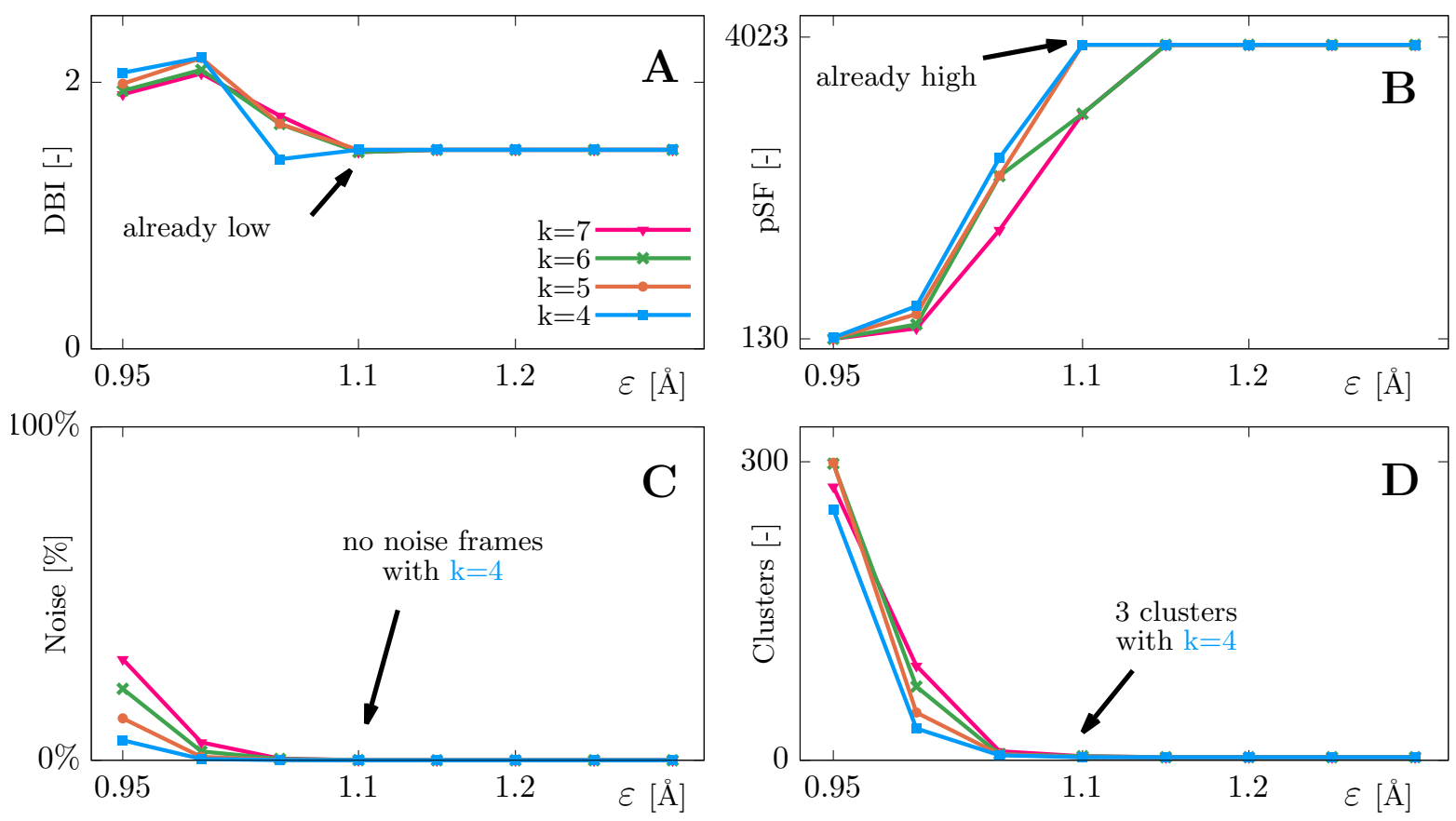

Figure 3: Clustering descriptors utilised for the tuning of the dbscan parameters: (A) the Davies-Bouldin index (DBI), (B) the pseudo-F statistic (psF), (C) the percentage of noise frames, and (D) the number of clusters. The descriptors were obtained by clustering the MD trajectory with benzene as the cosolvent and considering only the alpha carbon atoms for the RMSD calculations. The $\varepsilon$ parameter in units of Angströms are shown on the horizontal axes in all cases, while on the vertical axes the various unitless descriptors are shown. 
Since the clustering of the trajectories obtained with different solvents is carried out independently from each other, it is possible that some cluster representatives coming from different trajectories are quite similar to each other. This redundancy would clearly not be optimal as it increases the computational requirements of the ensemble docking calculations without providing much additional information. It is worth investigating this redundancy as its presence would indicate that the cosolvent simulations do not provide protein conformations different than those from the simulation in pure water. To this end, another clustering is performed utilising the parameters selected in the previous section, but with all trajectories considered at once. This clustering yields 18 cluster representative structures, which is only marginally less than the 19 ones obtained with the original clustering scheme. The fact that the clustering algorithm cannot merge many clusters coming from different solvent trajectories, i.e., returns a similar number of clusters as when the trajectories are considered individually, signals that the three trajectories with different solvents indeed visit markedly different conformations.

To further confirm the assumption that conformations coming from trajectories with different solvents are more dissimilar to each other than conformations coming from the same trajectory, the RMSD distances between all cluster representatives are calculated. More specifically, the 19 representative protein conformations obtained in the previous section are taken, and RMSD values between all possible pairs formed from them are calculated, considering only their alpha carbons. By looking at the probability distribution of these RMSD values for conformation pairs obtained from the same or from different MD trajectories, one can compare the intra- and intertrajectory similarity of protein conformations. Figure 4 shows this data grouped by the solvent pairs from which the protein conformations are obtained. For example, the solid cyan curve represent the distribution of RMSD values between protein conformations coming from the same water simulation, while the dashed yellow line represents the distribution of RMSD values between conformations from the water simulation and conformations from the benzene/water simulation. The most noticeable 
feature of this graph is that the intratrajectory distances are considerably smaller than the intertrajectory ones, with the solid curves being to the left of the dashed ones. There is only a single outlier benzene conformation, which is quite dissimilar to all other cluster representatives obtained from this trajectory. Therefore, the data represented in Figure 4 validates our assumption that by utilizing different solvents, a more diverse set of protein conformations can be obtained than in the case when only a single solvent (water) is employed.

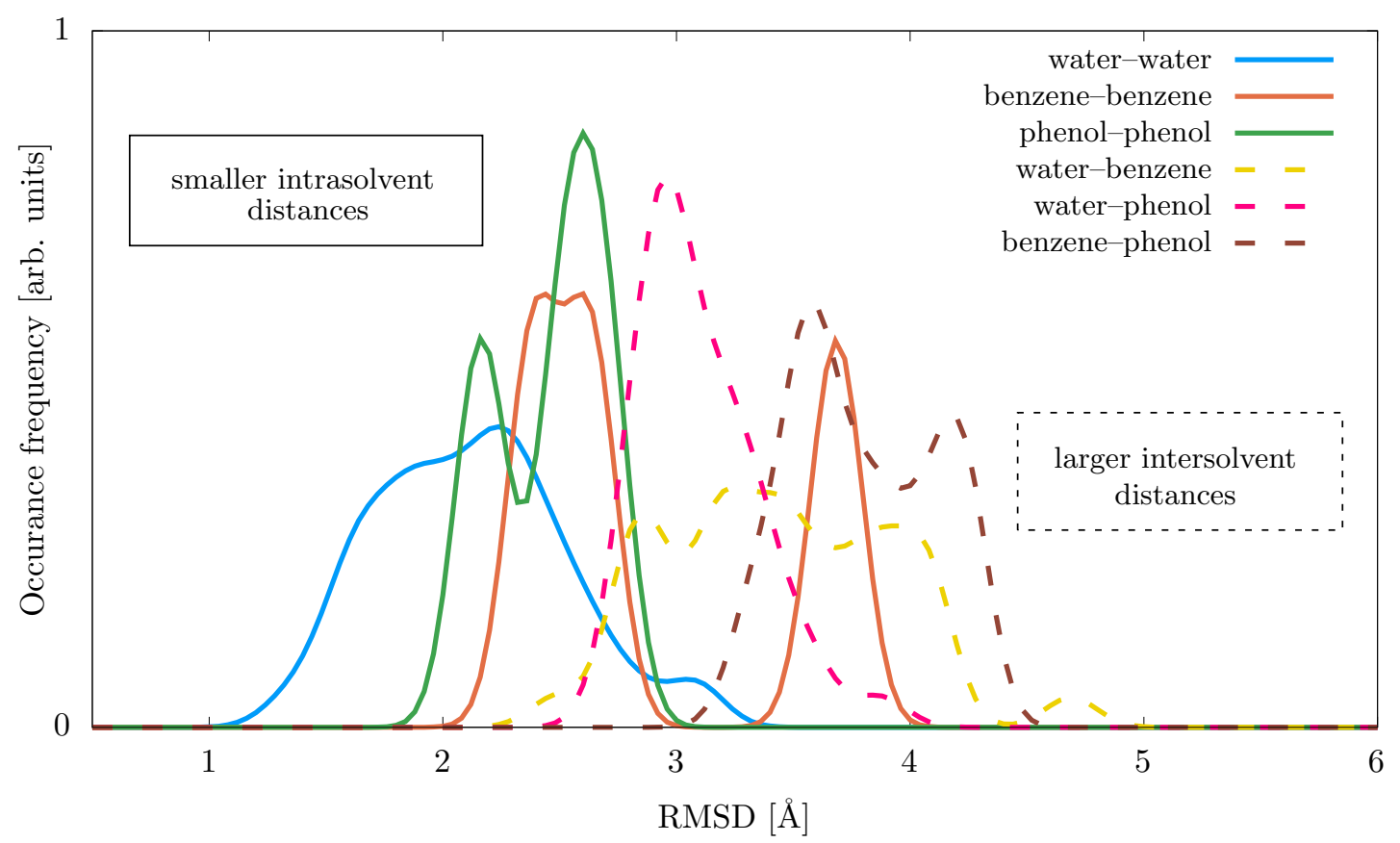

Figure 4: The distribution of the RMSD deviation distances between the selected protein conformation cluster representatives. The curves represent the frequencies with which a given RMSD value is found among all distances calculated between conformations coming from the trajectories denoted by their solvent in the legend.

\section{Performance of the Docking Protocol}

Before performing ensemble docking calculations with the obtained set of protein conformations, first the accuracy of the employed docking protocol is evaluated. To this end, a number of confirmed binders for the RdRp of SARS-CoV-2 are obtained from Reference 14, along with the most important interacting residues for each binder. To obtain these results, the authors utilised the experimentally determined apo protein structure with PDB 
code 6M71. To compare the performance of AutoDock Vina, we have performed docking calculations considering only the same crystallized structure. However, there are still significant differences in the preparation of the protein structure and the execution of the docking calculations between the cited work and the one presented here. Most notably, no minimisation of the structure is performed here, and all residues of the protein are considered rigid during the docking calculations, contrary to the work of Ahmad et al., where key residues around the active site were treated as flexible. Therefore, although perfect agreement with the previous simulations will not be achieved, it is expected that the published binders and their poses can be at least partially reproduced using the docking procedure adopted in this work.

The results obtained with AutoDock Vina and those from Reference 14 are compared in Table 1 for the seven ligands listed in the first column. This subset of the binders presented in the original article, comprises the ligands for which a 3D structure could be obtained from the ZINC database. In addition, the second column of the table shows the most important interacting residues reported for each of the binders. The results returned by the AutoDock Vina simulations performed by us are given in the last two columns of the table. Specifically, the rank of the best poses returned by Vina, where the binder interacts with the residues given in the second column, is reported. Moreover, in the fourth column, the total number of found poses - out of the 20 most important ones - which interact with the specified residues is given. It can be seen, that there are only two ligands (benzquercin and pegamotecan), out of the seven considered, for which AutoDock Vina does not find the pose reported in the literature. For these two ligands, a region near the active site of the protein is clearly favored by Vina. For the other five ligands, the pose predicted in the literature is found by Vina as well, and in three cases it is among the top five returned poses. Moreover, in the case of three ligands, the predicted interacting residues are reproduced by more than one pose, indicating the stability of these results. Since the main focus of this study is not on the accuracy of the docking calculations in itself, but rather on the investigation of the effects 
of cosolvents and protein dynamics on the docking results, the proposed docking procedure is deemed suitable and is, therefore, utilised throughout the rest of our work.

Table 1: Comparison of the results of AutoDock Vina to the binding poses reported in the literature. The data in the first two columns of the table is reproduced from Tables 1 and 2 of Reference 14, originally published by Ahmad et al.. The third and fourth columns show the rank of the best pose and the total number of poses returned by AutoDock Vina that interact with the residues shown in the second column.

\begin{tabular}{c|ccc}
\multirow{2}{*}{ ligand } & reported interacting residues & $\begin{array}{c}\text { rank of first } \\
\text { matching pose }\end{array}$ & $\begin{array}{c}\text { number of } \\
\text { matching poses }\end{array}$ \\
\hline \hline \multirow{2}{*}{ ornipressin } & $\begin{array}{c}\text { ASP760, THR591, ASP865, } \\
\text { GLN815, SER814, CYS813, } \\
\text { GLU811, TYR619 }\end{array}$ & 1 & $8(40 \%)$ \\
benzquercin & ARG553, LYS798 & - & $0(0 \%)$ \\
cisatracurium & ARG553, ARG555, GLU811 & 2 & $1(5 \%)$ \\
ditercalinium & ASP623, ASP760 & 9 & $1(5 \%)$ \\
examorelin & ASN691, HIS810 & 15 & $3(15 \%)$ \\
nacartocin & LYS798 & 4 & $3(15 \%)$ \\
pegamotecan & LYS621 & - & $0(0 \%)$
\end{tabular}

\section{Ensemble Docking Simulations}

\section{Binding Free Energy Criterion}

After the adeptness of the docking protocol has been verified, the distribution of the binding free energies obtained from docking the approximately 2000 FDA approved drugs to the 19 representative protein conformations is examined. The distributions of the best binding energies of each protein conformation-ligand pair are shown on Figure 5A, separately for the three types of MD trajectories. The most noticeable feature of this plot is that the distributions of the two cosolvent trajectories extend to lower binding energies (or larger binding energies in absolute value), than that of the simple water trajectory. Accordingly, the number of unique ligands with favorable binding energies are larger for the cosolvent trajectories as can be observed from the coloured numbers immediately to the left of each distribution 
on the figure. The presence of such general trend likely indicates some significant differences in the protein conformations utilised during the docking calculations. For example, it might happen that one or more binding regions, especially those containing hydrophobic residues, are more accessible to ligands in the conformations obtained from the cosolvent trajectories than in those obtained from the water solvated trajectory, and therefore, the binding energies are more favourable in the cosolvents. The larger druggability of some pockets when the nonpolar cosolvents are used in the simulations will be discussed in more detail later.

Since the binding energy of a given ligand is very strongly dependent on the conformation of the target protein, it is expected that some ligands bind strongly only to specific protein conformations, which are not present in all the solvent situations. In fact, it has been demonstrated on Figure 4 that the different solvent trajectories sample markedly dissimilar protein conformations. Consequently, it can be expected that some strong binders can only be found when the docking is performed with a specific solvent. When this is the case, then, one should observe that docking to one or even to just a few conformations is not sufficient to find all the best binding ligands for a given protein. This would also mean that utilising a well constructed ensemble of protein conformations during the docking calculations is beneficial because it aids the discovery of new binders and better binding poses. To test this hypothesis, the selection criterion for choosing the best binding ligands is set to -9.9 kcal/mol binding energy, as this arbitrary threshold selects sufficiently many ligands for each trajectory - around 100 ligands - from the 1957 initially docked in the protein, as can be seen in Figure 5A.

The binders selected in this way are subsequently grouped according to which cluster representative protein structures they bind to. Note that a ligand can be in multiple groups simultaneously if its binding energy is lower than $-9.9 \mathrm{kcal} / \mathrm{mol}$ for more than one protein conformation. Next, the cluster representatives are ordered in descending order according to the number of ligands they bind. Finally, the representative protein conformation with the highest number of binding ligands is selected, and its ligands are removed from the lists 
of all other conformations, which happen to also bind that ligand. This last step is repeated until all conformations and all binding ligands are accounted for. From the data obtained in this manner, cumulative ligand binding plots are created and displayed on Figure 5B,C. These diagrams visualise the number of protein conformations which are necessary to find a certain percentage of all the best binding ligands, discovered through any of the cluster representatives. On Figure 5B, the ligands discovered by different solvent trajectories are separated, and the cluster representatives are ordered on the horizontal axis in decreasing importance (from many to few new ligands discovered). It can be observed that with a single protein conformation, only about $75 \%$ of all binders of the cosolvent trajectories and about $40 \%$ of the binders of the water solvent trajectory would have been discovered, even if the conformation with the most binders would have been utilised. Moreover, all protein conformations have unique ligands that only bind to them, and not to other conformations of the given trajectory. These results, therefore, validate the increased computational costs of docking to an ensemble of protein structures, as they show that significantly more binders can be discovered by utilising multiple protein conformations than in the case of a single considered structure.

Figure 5C shows a similar curve but, instead of considering the ligands of the different protein conformations separately, they are merged together for each solvent. In this case, the horizontal axis displays the various solvents that the conformations were obtained from. From this plot it can be observed that every MD trajectory for a specific solvent is able to find unique ligands that do not bind to conformations of the other trajectories, further validating the use of multiple solvents. The last data point of this plot corresponds to the binders discovered by docking to the apo crystal structure of the protein. It is clear that no new ligand is found by docking to this structure, that would not have been already found by one of the conformations obtained from the MD trajectories. This observation indicates that the trajectories sample a wide range of conformations, and can even account for those ligands that would bind to the crystallised protein structure. 


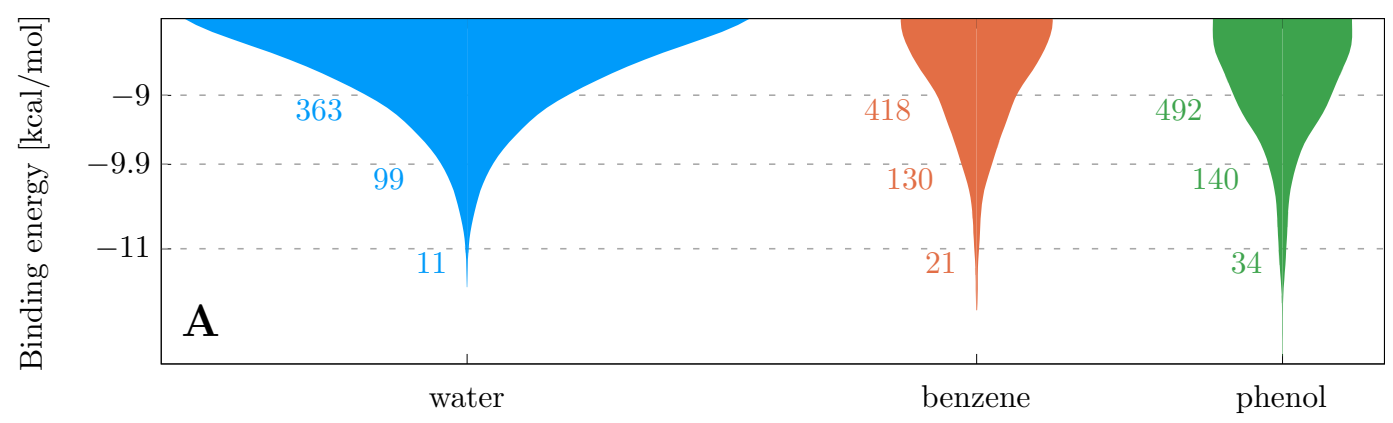

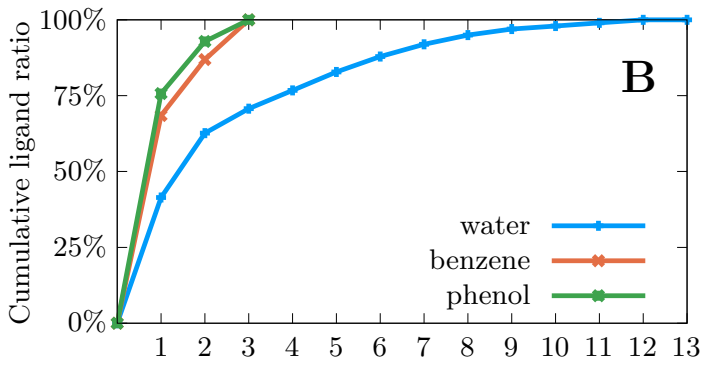

Index of representative confromation

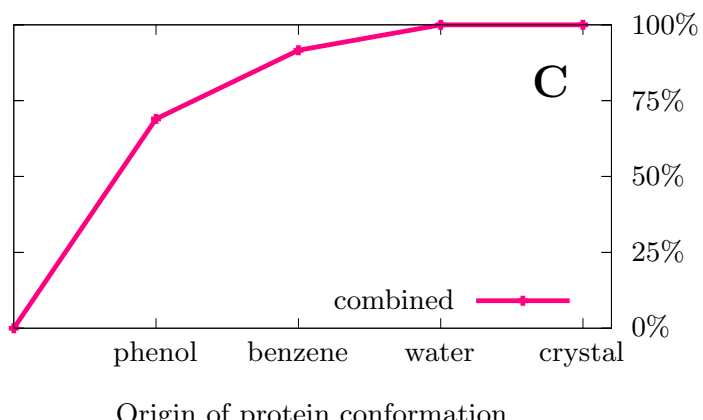

Origin of protein conformation

Figure 5: (A) The distribution of the ligand binding energies as calculated by AutoDock Vina. The numbers to the left of the distributions indicate the number of unique ligands which have a binding energy lower than the given value for at least one of the conformations obtained from that solvent. Note that the distribution widths cannot be directly compared. (B) Cumulative ratio of the ligands found if only a subset of the protein conformations from each trajectory is considered for docking. (C) Cumulative ratio of the ligands discovered by considering each trajectory or the apo crystal structure. The cluster representatives or trajectories on the horizontal axes are ordered in decreasing importance, from most to least new ligands discovered. 


\section{Binding Free Energy Gap Criterion}

A common approach to selecting the most promising binders from the candidate ligands in a VS campaign is to consider the binding free energy estimate calculated by the docking program, as was done in the previous section. However, the inaccuracies of the binding energy estimates calculated by such programs are well known. ${ }^{90}$ Additionally, it is noted that this binding energy is naturally dependent on the size of the ligand being considered. Since ligands with a high number of atoms can benefit from more interactions between their atoms and those of the protein, this metric makes the selection of ligands that contain only a small number of atoms very unfavorable interacting moieties. These imperfections of the binding energy estimate make it a somewhat unreliable criterion for the selection of the best binding ligands. To devise an improved metric of the ligand interaction strength, one commonly used idea is to correct for the entropic terms missing from most scoring functions, by accounting for the spatial distribution of the docked poses. ${ }^{91,92}$ However, in the case of AutoDock Vina, the obtained best poses are first clustered by the program, and only the cluster representative poses are returned, ${ }^{56}$ which makes the above mentioned pose distribution based corrections unfeasible. In this section, an alternative approach to enhance the selection of the best binders is devised and evaluated, that can be carried out relying only on the information provided by AutoDock Vina.

Our first attempts towards devising such a ligand selection criterion revolved around scaling the binding energy estimate by the number of heavy atoms in the considered ligand. Unfortunately, this approach turned out to favor small ligands too much, while the inclusion of some parameter to scale this bias down, seemed too arbitrary. Consequently, the procedure presented here is based solely on the binding energies themselves. More specifically, it evaluates the binding free energy gap between the two best poses found for the ligand. The idea behind this approach is that a truly good docked pose should be something out of the ordinary compared to the myriad of other suboptimal poses. Given the scarcity of such extraordinary minima of the scoring function, it is expected that only a small fraction 
of the stochastic optimisation runs, performed during a docking calculation, will find the corresponding ligand poses. In practice, this phenomenon could manifest itself in the form of a single docked pose with a highly desirable binding energy estimate, found among the many other mediocre poses in the results of a docking calculation. A large gap between the binding energies of the two best docked poses for a given ligand could indicate such a situation and consequently signal a true binder of the target protein. Therefore, it is proposed to categorise ligands as binders (non-binders) based on whether the gap between their two best binding energies is larger (smaller) than a given threshold. Finally, it is possible that this criterion functions best in combination with a simple binding energy threshold, where it would serve to eliminate some large ligands that are only categorised as binders due to the bias of the latter criterion towards larger ligands.

As the first step of evaluating the performance of this new ligand selection criterion, its preferred binders are compared to those selected based on their binding energy. If very few or no common ligands are found between the two criteria, it could indicate that this alternative selection method does not perform as expected, because even though the binding energy estimate provided by the docking program is an imperfect one, in general it will select reasonable ligands. To carry out this comparison, two sets of selection thresholds were determined for the binding energy and energy gap criteria each. The thresholds in these sets were constructed separately for the protein conformations coming from different solvent trajectories, such that they select approximately 100 ligands for each trajectory. This results in thresholds between -8.8 and $-10.2 \mathrm{kcal} / \mathrm{mol}$ for the binding free energy and between 0.6 and $1.2 \mathrm{kcal} / \mathrm{mol}$ for the binding free energy gap. With the best binders selected separately by the two different criteria, the ratio of common ligands can now be defined to be:

$$
R=\frac{\left|S_{1} \cap S_{2}\right|}{\max \left(\left|S_{1}\right|,\left|S_{2}\right|\right)},
$$

where $S_{1}$ and $S_{2}$ are the sets of ligands selected by one of the criteria. On Figure 6A, this 
ratio of ligands selected by both criteria is plotted separately for the protein conformations obtained from each MD trajectory and the crystal structure. One can observe that about 15-20\% of the best 100 ligands are common to both criteria. Even though the two criteria select ligands in entirely different manners, at least part of the ligands they favor are common to both of them. This gives us some confidence that the energy gap based selection criterion can indeed be suitable to augment and complement simple binding energy based selection methods.

After observing that a substantial ratio of the selected ligands are common for the two selection criteria, the question naturally arises: are these common ligands especially suitable binders? This would validate the use of the composite ligand selection method involving the binding energy and binding energy gap criteria. Therefore, it would be beneficial to confirm that the ligands selected based on the composite selection criterion exhibit a favorable binding energy as well, thus further increasing the confidence in the adeptness of this ligand selection method. To investigate this, the normalised distribution of the binding energies are plotted for the ligands present in the top 100 binders using the binding energy criterion and for the ligands which satisfy both the binding energy and energy gap criteria. The first distribution is represented by the lightly coloured left half of each violin plot of Figure 6, while the second distribution is represented by the violin plots with a darker color. The figure clearly shows that the binding energies of those ligands which are common to both selections are lower (more favorable) than those of ligands selected solely by their binding energy. It is therefore confirmed that ligands satisfying both criteria tend to bind more favourably than ligands which satisfy only the binding energy criterion.

\section{Binding Sites of the Target Protein}

In this section, the binding sites of the target protein are identified and characterised through the ligands interacting with them. To this end, a number of promising ligands are selected 

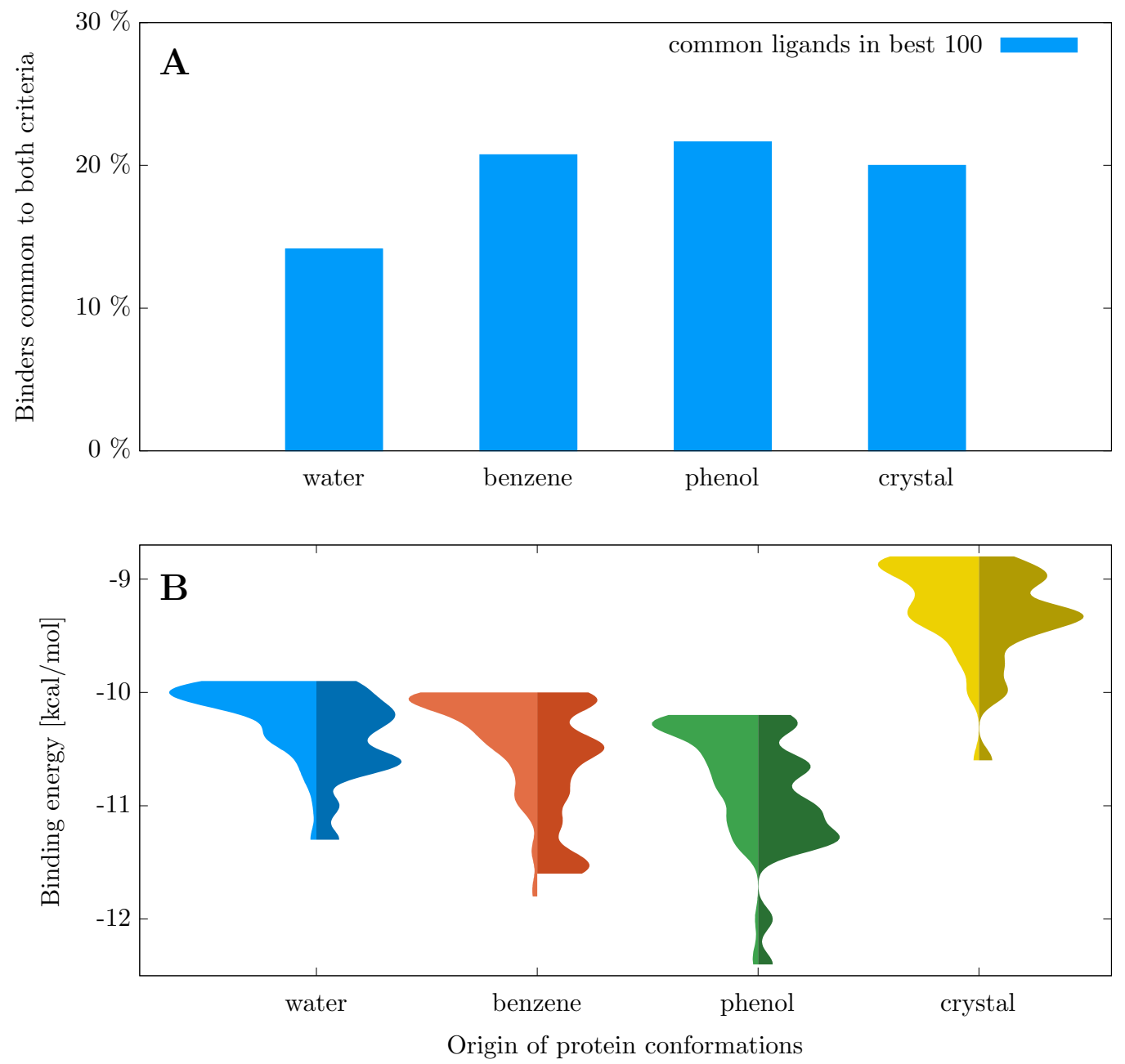

Figure 6: (A) The ratio of ligands selected both based on their binding free energy and the binding free energy gap between their two best poses. Ligands binding to conformations coming from different solvent trajectories (or to the crystal structure) are treated separately. For the exact definition of the ratio plotted here, see Equation 1. (B) Comparison of the binding energy distributions for the ligands selected based on solely their binding energy and for the ligands selected both based on their binding energy and energy gap between their two best poses. The distributions are plotted separately for the ligands of the three solvent trajectories and the crystal structure. The left half of each violin plot represents the distribution obtained for the ligands selected by their binding energy, while the right half denotes the same distribution for the ligands selected by both their binding energy and energy gap. 
with the previously discussed two selection criteria. In both cases, the selection threshold is determined in a way to yield approximately 100 ligands for the water solvent MD trajectory. Then, the same threshold is employed for the cosolvent simulations. For this purpose, a binding energy threshold of $-9.9 \mathrm{kcal} / \mathrm{mol}$, and an energy gap threshold of $1.2 \mathrm{kcal} / \mathrm{mol}$ were found to be the most optimal.

With the binders selected in this way, the most important interacting sites of the protein can be identified by visual inspection of the most favorable docked poses of the best binders. These poses are visualised on Figure 7, considering the ligands of both selection criteria together. After inspecting the docked ligands of the nine most important binding sites, interacting protein residues have also been assigned to them, collected in Table 2. It is noted that binding site number 4 identified here corresponds well to the active site of the protein, as reported in Reference 14. Therefore, the interacting residues shown for this pocket in Table 2 are taken from that work.

Table 2: The interacting residues of the binding pockets discovered through ensemble docking. The interacting residues of the active site, which corresponds to the fourth pocket in the numbering of this work, are taken from Section 1.1 of Reference 14 by Ahmad et al. The chain identifiers and residue numbers are consistent with the numbering of the structure with PDB identifier $6 \mathrm{M} 71 .{ }^{74}$

\begin{tabular}{c|c} 
pocket nr. & interacting residues \\
\hline \hline 1 & chain A: LEU172, TYR265, THR319, PRO323, THR394, PHE396, LEU460 \\
2 & chain A: ASP36, TYR38, ILE66, SER68, ASP208 \\
3 & chain A: LEU49, ASP711, ASP714, GLN773 \\
& chain A: ASP618, CYS622, ASN691, ASN695, MET755, ILE757, LEU758, \\
4 & SER759, ASP760, ASP761, ALA762, VAL763, GLU811, PHE812, CYS813, \\
& SER814 \\
5 & chain A: ASN447, chain B: PRO133, ASP134, TRP182, PRO183, chain \\
& B: LYS27 \\
7 & chain A: ASN414, ASN416, ASP418, VAL844, chain C: ILE68, chain D: \\
7 & ARG111 \\
9 & chain B: TYR138, THR145, TRP154, GLU155, LEU169 \\
& chain A: GLU254, ASP269, LYS272, ARG285
\end{tabular}




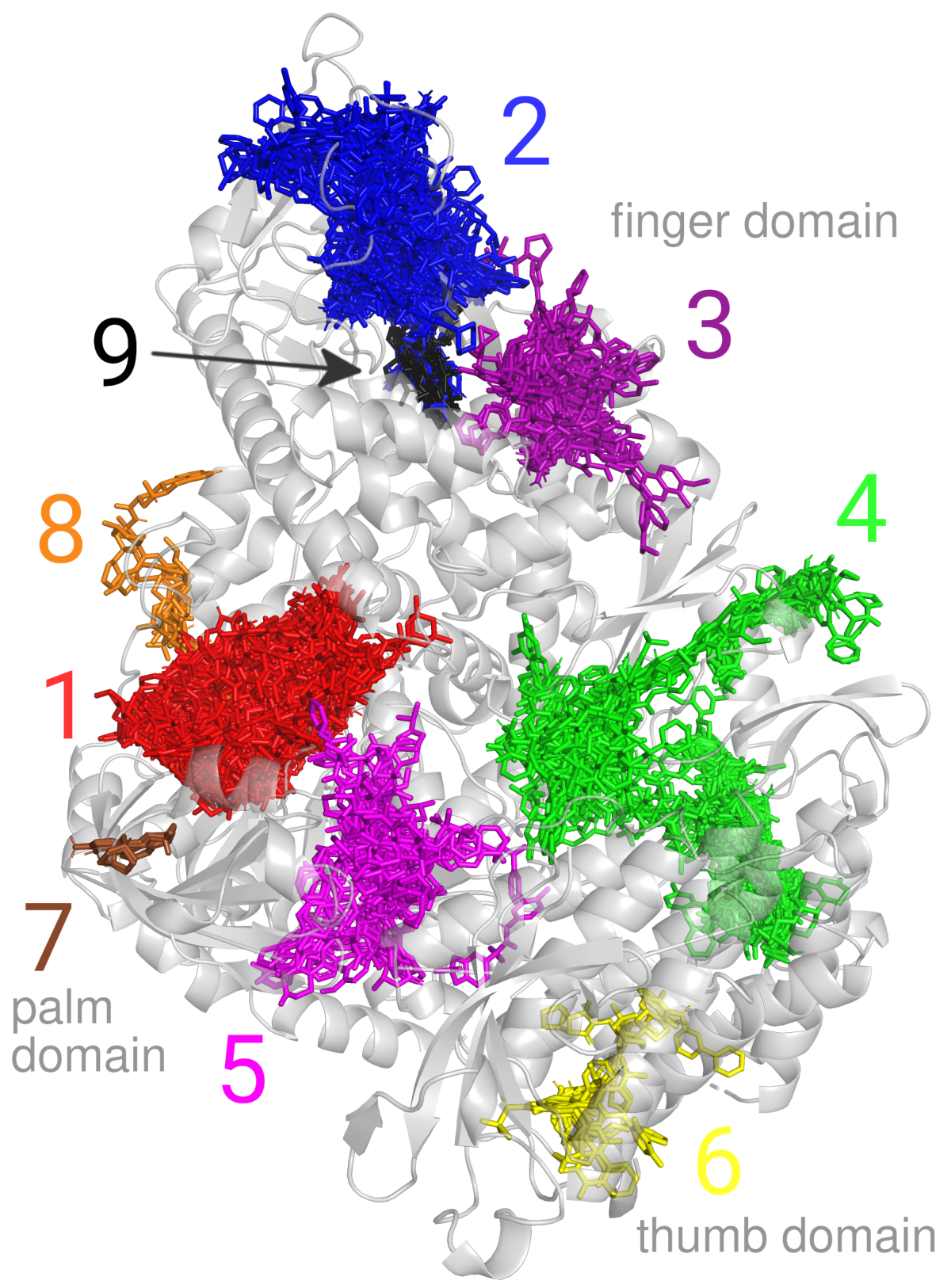

Figure 7: Binding sites on the protein, as identified by the visual inspection of the poses of the best binding ligands. The protein structure is the first cluster representative structure obtained from the water solvent MD trajectory. The coloured molecule clusters are the superimposed binders for all cluster representative protein structures as selected by either one of the binding energy criteria. Note that only those ligands are shown which bind to one of the identified pockets, an additional $2 \%$ of the binders, which bind to other regions of the protein are hidden. 
After the binding sites of the protein have been identified, the number of ligands binding to each of them are counted and presented in Table 3, separately for the MD trajectories with different solvents, and for the apo crystal structure, and considering the binding energy and binding energy gap criteria as selection methods. Overall, the nine identified pockets are responsible for $97-99 \%$ of the best binders found for every trajectory, which clearly highlights the importance of these regions of the protein surface for potential subsequent targeted VS campaigns. It is most interesting that some of the identified pockets only bind ligands if specific cosolvents or even a specific ligand selection method is employed. This observation shows how the presence of cosolvent probes significantly influence the protein structure, so much so that it can lead to the opening or the closing of certain pockets. Regarding the population of the active site (pocket 4), on the one hand, it is reassuring to see that multiple binders are found, with all three solvents considered, as this site has already been identified as druggable. ${ }^{14-17}$ On the other hand, no binders are found for this site if the crystallised, apo protein structure is employed in the docking calculations. This fact emphasises the importance of protein flexibility during ligand uptake, and makes it clear why docking to a single, crystallised, apo protein structure can be inadequate to utilise the full power of docking calculations and VS.

Considering now the other binding sites of the protein, the most highly populated pocket is either the first or the second one, depending on the cosolvent and binder selection criterion employed. Among these, the first identified pocket is especially promising, as ligands bound here tend to be very well buried in the protein (see Figure 7). The fact that these two pockets are populated by a relatively large number of ligands, regardless of the solvent utilised to obtain the protein conformations, signals that they are quite stable and are open in most of the conformations regularly visited by the protein. On the other hand, the sixth and seventh pockets are almost exclusively populated with a few ligands when protein structures obtained from the benzene cosolvent MD trajectories are considered. It seems logical that the cause of this phenomenon is that the benzene probes were able to induce some conformational local 
changes in these regions of the protein. These latter two pockets are therefore more likely to be cryptic pockets, open only in rarely visited protein conformations or in the presence of binders.

For the third pocket, similar results are found as for the sixth and seventh ones, with the exception that some ligands are also selected for the conformations obtained from the phenol cosolvent MD trajectory. The fact that on top of the benzene cosolvent molecules, the more polar phenol probes were also able to open this pocket to a certain degree, could be connected to the higher ratio of hydrophilic residues around this binding site (see Table 4). Finally, the fifth, eighth and ninth pockets all seem to be more open in the conformations coming from the water solvent trajectory, with the latter two only having ligands if the energy gap ligand selection criterion is considered. As can be seen in Table 4, the residues around these pockets also tend to be more hydrophilic than in the case of some other sites. Remarkably, these pockets are also not present in the crystallised apo structure. It is therefore very likely that some reorganisation of the neighbouring residues, accounted for during the conventional MD calculations with water as the solvent, are necessary for their opening.

Table 3: The distribution of the binders, as selected by the two ligand selection criteria, across the various binding sites of the protein. Ligand counts are reported separately for each cosolvent utilised to obtain the protein conformation to which the ligand binds and the apo crystal structure. For the definition of the pocket numbering see Figure 7 and Table 2.

\begin{tabular}{c|cccc|cccc}
\multirow{2}{*}{ pocket } & \multicolumn{4}{|c|}{ binding energy criterion } & \multicolumn{4}{|c}{ energy gap criterion } \\
& water & benzene & phenol & crystallized & water & benzene & phenol & crystallized \\
\hline \hline 1 & 56 & 8 & 99 & 2 & 31 & 3 & 9 & 1 \\
2 & 9 & 67 & 22 & 1 & 25 & 13 & 11 & 0 \\
3 & 0 & 11 & 4 & 3 & 0 & 3 & 2 & 1 \\
4 & 4 & 29 & 9 & 0 & 3 & 10 & 2 & 0 \\
5 & 29 & 2 & 5 & 0 & 19 & 0 & 0 & 0 \\
6 & 0 & 6 & 0 & 0 & 1 & 7 & 0 & 1 \\
7 & 0 & 4 & 0 & 0 & 0 & 5 & 0 & 0 \\
8 & 0 & 0 & 0 & 0 & 4 & 2 & 0 & 0 \\
9 & 0 & 0 & 0 & 0 & 8 & 0 & 0 & 0 \\
other & 1 & 3 & 1 & 0 & 5 & 0 & 0 & 0 \\
\hline total & 99 & 130 & 140 & 6 & 96 & 43 & 24 & 3
\end{tabular}


Next, it is assessed whether or not the polarity of the cosolvent molecules correlate with the hydrophilicity of the binding pockets that are open during the MD trajectory calculated with that cosolvent. To perform this analysis, the residues nearest to the nine binding sites identified in the previous section are selected. This is done by first selecting the protein conformation for which the highest number of binders are found for the pocket in question. Then, those residues which have atoms not farther than $3 \AA$ from one of the atoms of one of the binders are chosen. Then, the selected protein residues are categorised as either hydrophilic or hydrophobic based on the results presented in Reference 93. The ratio of hydrophobic residues for each pocket, along with the cosolvent with which the most populated protein conformation is obtained for that pocket, is shown in Table 4. It is noted that other methods for selecting the nearest residues for each binding sites have been experimented with (e.g., defining a center for each pocket and defining a distance threshold from that point), and these methods yielded very similar results to those presented here.

Table 4: The ratio of hydrophobic residues near each pocket, along with the cosolvent with which the protein conformation which produced the most binders for that pocket was obtained. The assignment of residues to each pocket is discussed in the main text.

\begin{tabular}{c|ccc} 
pocket & total no. of residues & hydrophobic residues [\%] & cosolvent \\
\hline \hline 1 & 34 & 47 & phenol \\
2 & 35 & 34 & benzene \\
3 & 14 & 21 & benzene \\
4 & 19 & 58 & benzene \\
5 & 26 & 31 & water \\
6 & 9 & 55 & benzene \\
7 & 6 & 50 & benzene \\
8 & 4 & 0 & water \\
9 & 9 & 33 & water
\end{tabular}

Looking at the hydrophobicity data in Table 4, it is apparent that the pockets which were most open in the protein conformations coming from the water solvent trajectory all have low hydrophobic residue ratios. In particular pockets 8 and 9 , which were previously seen to bind ligands almost exclusively if protein conformations from the water solvated 
trajectory are considered (see Table 3), have a low ratio of hydrophobic residues. On the contrary, the most apolar cosolvent, benzene, opens the most hydrophobic pockets best. More specifically, pockets 6 and 7 , which were only discovered with the benzene cosolvent protein conformations, both have a hydrophobic residue ratio of at least $50 \%$. Pockets 2 and 3 are also most populated with the benzene cosolvent protein conformations, even though both of them are decidedly hydrophilic in character. However, it is worth noting that these pockets are also well populated when protein structures from trajectories with water and especially with phenol are considered. Based on the above results, a link between the polarity of the solvent, and the hydrophilicity of the pockets opened by said solvent can be confidently established. This highlights once again the usefulness of employing cosolvent MD simulations for the generation of protein conformations in ensemble docking: by utilising apolar cosolvent probes, new, more hydrophobic binding sites can be opened that would otherwise not be discovered.

Finally, the volumes of a stable and a transient pocket across conformations of the ensemble are compared to identify further characteristics of the different pocket types. The pocket volumes are calculated by mdpocket and are shown on Figure 8 for all 19 protein conformations. Looking at the top plot of this figure, where the volumes of the transient pocket 7 are shown, one can observe that the structures obtained from the benzene MD trajectory harbor pocket conformations with the largest volumes, in one case reaching more than 400 $\AA^{3}$. This observation is to some extent in line with the fact that ligands are only able to bind to this pocket when the protein conformations obtained from the benzene trajectory are considered (see Table 3). In general, it is clear that this pocket has a non-zero volume only in a small subset of the complete conformational ensemble, further confirming its transient nature. Furthermore, the ability of the benzene cosolvent simulations to provide open pocket conformations is again demonstrated. It must be noted that mdpocket calculates non-zero volumes for some protein conformations obtained from the water solvent trajectory as well, indicating the presence of an open pocket in those conformations. However, the docking 
calculations found no ligands for the water solvent trajectory, not even for these conformations. This indicates that simple descriptors, such as the volume, although valuable, are not perfectly reliable and cannot be considered a substitute to the more accurate explicit docking calculations. In other words, the ability of a pocket to accommodate binders cannot be quantified by just looking at simple structural descriptors.
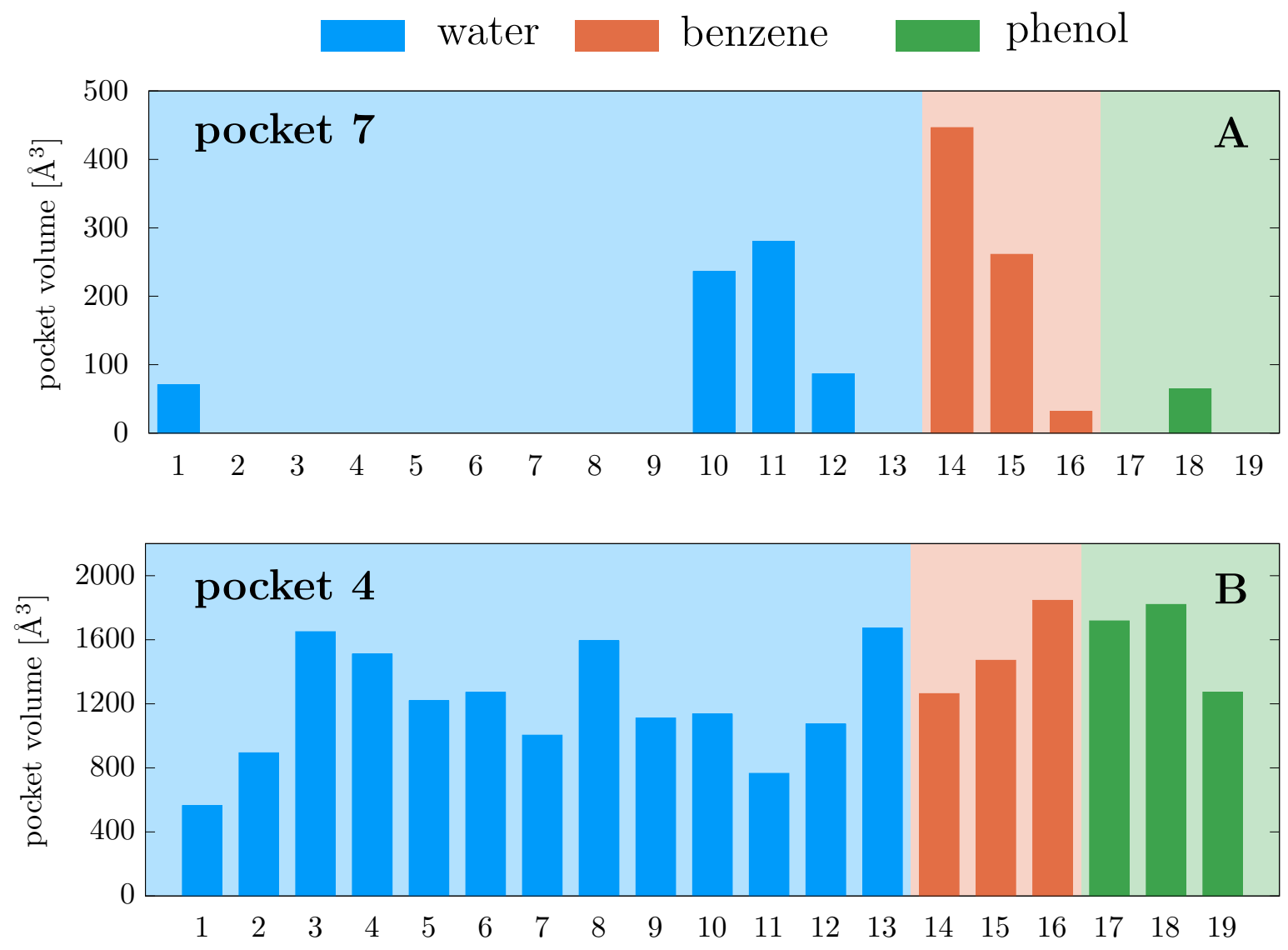

Figure 8: Volumes of pocket 4 (A) and 7 (B) calculated for all considered protein conformations. Pocket volumes were calculated with the mdpocket program. For the definition of these binding pockets, see Table 2 and Figure 7.

In comparison to the results found for pocket 7 , one can observe a more homogeneous pocket volume distribution across the various protein structures for the stable pocket 4 . However, the fluctuation between protein conformations is still large with the volume of the active site pocket ranging between 600 and $1700 \AA^{3}$. Comparing the two binding sites further, pocket 4 is found to have a much larger volume than the transient pocket 7 in all frames. The 
main similarity between these pockets is that their largest respective volumes are calculated when conformations coming from the benzene cosolvent trajectories are considered. However, this is not surprising because both pockets present the largest number of binders for the benzene/water simulation, as was shown in Table 3. That being said, the difference in pocket volume between the water solvent and benzene cosolvent or between the phenol and benzene cosolvents trajectory frames is far less significant in the case of the active site. This agrees with the fact that several binders are found to bind pocket 4 for the water and phenol/water trajectories, while this is not the case for pocket 7 , which is populated only when benzene is used as cosolvent. The ability of cosolvent MD trajectories to provide higher pocket volumes in comparison to traditional MD simulations for both stable and transient pockets is worth mentioning.

\section{Conclusions}

In drug discovery projects, the technique of VS has proved to be especially useful, contributing to the discovery of hundreds of small molecule drug candidates by performing docking calculations to a given protein structure with thousands of ligands. Owing to its numerous successes, considerable efforts are expended to achieve further improvements in its accuracy and usability. For example, the introduction of conformational sampling has been shown to be crucial to improve the efficacy of VS campaigns. In the present work, we have considered conformational sampling by performing ensemble docking calculations on different protein geometries generated by classical MD simulations. To further improve the sampling of the configurational space, the simulations were evolved in three different solvent scenarios: on top of the usual water solvent, benzene/water and phenol/water mixtures were utilised. Due to the COVID-19 pandemic that have already claimed millions of lives around the world, the simulations were performed for the RdRp protein of the SARS-CoV-2 virus as target of almost 2000 FDA approved drug molecules, in the hopes of revealing information, especially 
from the methodological point of view, that can contribute to the development of effective treatments.

The ensemble of protein geometries employed in the docking calculations were generated by density based clustering of the MD trajectories evolved in the three solvents. The clustering analysis provided 13, 3 and 3 representative conformations for the simulations in water, benzene/water and phenol/water, respectively. These cluster representative frames selected from different cosolvent trajectories proved to harbor protein conformations with meaningful differences, as evidenced by their large RMSD distances from each other. This result in itself highlights the importance of running MD simulations with different cosolvents since the different protein conformations obtained could accommodate different binders compared to the traditional dynamics simulations run in pure water as the solvent.

Ensemble docking calculations were performed utilising the 19 representative protein conformations and the crystal structure, together with a set of approximately 2000 FDA approved drug molecules. The best binders were identified attending to two different selection methods based on the binding free energy and the binding free energy gap between the two best docked poses of a particular drug. The second selection method is intended to help in finding the ligands with the best interacting chemical moieties, regardless of the size of the ligand since the simple binding energy criterion might overestimate the importance of large ligands. It was found that both approaches select a reasonable ratio of common ligands. Most importantly, these ligands selected by both methods presented the most favorable binding energies of all the considered ligands. Therefore, the importance of using different binder selection methods - and a combination of them - to propose promising protein binder candidates was demonstrated.

Subsequently, utilising either of the two selection criteria, the best binders of the protein were selected. By inspecting the poses of these ligands, nine important binding sites (including the already known active site) of the protein were identified, that harbored the best pose for over $98 \%$ of the best binders. The pocket search was dependent on the solvent 
and on the binder selection criteria, indicating once again that the combination of different approaches can provide better results in VS campaigns. In addition, the importance of conformational sampling was evidenced, since only four pockets were identified by running the docking simulations employing the crystal apo structure of the protein, instead of the 19 representative conformations. By analysing the populations of the binding pockets across different MD trajectories they were separated into two groups, corresponding to stable and transient binding sites. It was found that stable pockets are open in many or all frames of the various MD trajectories, and they bind a relatively large number of ligands. On the contrary, transient or cryptic binding sites were found to be open only in a limited set of protein conformations, often originating from the same cosolvent MD trajectory calculated with an apolar cosolvent. In fact, a correlation between the polarities of the pockets and solvents were found.

In order to understand the effects of cosolvents better, the protein conformations around two selected binding sites were compared between representative structures originating from different MD trajectories. One of these selected pockets was the active site of the protein, which was characterised as a stable pocket, and the other one was a transient pocket distal to the active site. Considering first the transient pocket, it was found that only the apolar cosolvent benzene was able to induce conformational changes which lead to an increase of the pocket volume, and therefore to the opening of the binding site. Looking at the active site, it was revealed that this region of the protein is more conserved, since a more homogeneous pocket volume distribution across the protein structures and the solvents was observed. However, despite this relatively constant volume behaviour for the active site, it was found that the effects of cosolvents are significant on both cryptic and well known stable pockets, with more druggable protein conformations visited during cosolvent MD simulations than either those found in the crystallised protein structure or those visited during traditional MD trajectories with water as the solvent.

In summary, the use of dynamics simulations that introduce nuclear motion, the intro- 
duction of different solvent combinations that further improve sampling, and the application of different criteria to select the best binders from docking calculations provided a larger variety of potential binding sites and drug candidates for the SARS-CoV-2 RdRp protein than static docking calculations. It is seen that the combination of a wide range of dynamics and analysis techniques can significantly improve the efficacy of future VS projects aimed to search for drug candidates to fight against COVID-19 and other diseases.

\section{Acknowledgements}

We would like to thank Jeremy Harvey for very fruitful discussions. This work was funded by the Comunidad de Madrid through the Attraction of Talent Program (Grant Ref. 2018T1/BMD-10261). The Centro de Computación Científica of Universidad Autónoma de Madrid is thanked for computational resources.

\section{References}

(1) Durrant, J. D.; Urbaniak, M. D.; Ferguson, M. A. J.; McCammon, J. A. ComputerAided Identification of Trypanosoma brucei Uridine Diphosphate Galactose 4'Epimerase Inhibitors: Toward the Development of Novel Therapies for African Sleeping Sickness. Journal of Medicinal Chemistry 2010, 53, 5025-5032.

(2) Durrant, J. D.; Cao, R.; Gorfe, A. A.; Zhu, W.; Li, J.; Sankovsky, A.; Oldfield, E.; McCammon, J. A. Non-Bisphosphonate Inhibitors of Isoprenoid Biosynthesis Identified via Computer-Aided Drug Design. Chemical Biology $\&$ Drug Design 2011, 78, 323-332.

(3) Li, X.; Zhang, X.; Lin, Y.; Xu, X.; Li, L.; Yang, J. Virtual Screening Based on Ensemble Docking Targeting Wild-Type p53 for Anticancer Drug Discovery. Chemistry \& Biodiversity 2019, 16, e1900170. 
(4) Li, C.; Xu, L.; Wolan, D. W.; Wilson, I. A.; Olson, A. J. Virtual Screening of Human 5-Aminoimidazole-4-carboxamide Ribonucleotide Transformylase against the NCI Diversity Set by Use of AutoDock to Identify Novel Nonfolate Inhibitors. Journal of Medicinal Chemistry 2004, 47, 6681-6690.

(5) Cosconati, S.; Hong, J. A.; Novellino, E.; Carroll, K. S.; Goodsell, D. S.; Olson, A. J. Structure-Based Virtual Screening and Biological Evaluation of Mycobacterium tuberculosis Adenosine 5'-Phosphosulfate Reductase Inhibitors. Journal of Medicinal Chemistry 2008, 51, 6627-6630.

(6) Mullarky, E. et al. Identification of a Small Molecule Inhibitor of 3-phosphoglycerate Dehydrogenase to Target Serine Biosynthesis in Cancers. Proceedings of the National Academy of Sciences of the United States of America 2016, 113, 1778-1783.

(7) Cosconati, S.; Forli, S.; Perryman, A. L.; Harris, R.; Goodsell, D. S.; Olson, A. J. Virtual Screening with AutoDock: Theory and Practice. Expert Opinion on Drug Discovery 2010, 5, 597-607.

(8) Overington, J. P.; Al-Lazikani, B.; Hopkins, A. L. How Many Drug Targets are there? Nature Reviews. Drug Discovery 2006, 5, 993-996.

(9) Max Roser, E. O.-O., Hannah Ritchie; Hasell, J. Coronavirus Pandemic (COVID-19). Our World in Data 2020, https://ourworldindata.org/coronavirus [Online; accessed on 29th July 2021].

(10) Rathi, H.; Burman, V.; Datta, S. K.; Rana, S. V.; Mirza, A. A.; Saha, S.; Kumar, R. Review on COVID-19 Etiopathogenesis, Clinical Presentation and Treatment Available with Emphasis on ACE2. Indian Journal of Clinical Biochemistry 2021, 36, 3-22.

(11) Indari, O.; Jakhmola, S.; Manivannan, E.; Jha, H. C. An Update on Antiviral Therapy Against SARS-CoV-2: How Far Have We Come? Frontiers in Pharmacology 2021, 12, 133. 
(12) Rezagholizadeh, A.; Khiali, S.; Sarbakhsh, P.; Entezari-Maleki, T. Remdesivir for Treatment of COVID-19; an Updated Systematic Review and Meta-analysis. European Journal of Pharmacology 2021, 897, 173926.

(13) Beigel, J. H. et al. Remdesivir for the Treatment of Covid-19 - Final Report. The New England Journal of Medicine 2020, 383, 1813-1826.

(14) Ahmad, J.; Ikram, S.; Ahmad, F.; Rehman, I. U.; Mushtaq, M. SARS-CoV-2 RNA Dependent RNA polymerase (RdRp) - A Drug Repurposing Study. Heliyon 2020, 6, e04502-e04502.

(15) Ao, S.; Han, D.; Sun, L.; Wu, Y.; Liu, S.; Huang, Y. Identification of Potential Key Agents for Targeting RNA-Dependent RNA Polymerase of SARS-CoV-2 by Integrated Analysis and Virtual Drug Screening. Frontiers in Genetics 2020, 11, 581668-581668.

(16) Ruan, Z.; Liu, C.; Guo, Y.; He, Z.; Huang, X.; Jia, X.; Yang, T. SARS-CoV-2 and SARS-CoV: Virtual Screening of Potential Inhibitors Targeting RNA-dependent RNA Polymerase Activity (NSP12). Journal of Medical Virology 2021, 93, 389-400.

(17) Kandeel, M.; Kitade, Y.; Almubarak, A. Repurposing FDA-Approved Phytomedicines, Natural Products, Antivirals and Cell Protectives against SARS-CoV-2 (COVID-19) RNA-Dependent RNA Polymerase. PeerJ (San Francisco, CA) 2020, 8, e10480e10480.

(18) Cozac, R.; Medzhidov, N.; Yuki, S. Predicting Inhibitors for SARS-CoV-2 RNADependent RNA Polymerase Using Machine Learning and Virtual Screening. arXiv:2006.06523, 2020.

(19) Koulgi, S.; Jani, V.; Uppuladinne, M.; Sonavane, U.; Nath, A. K.; Darbari, H.; Joshi, R. Drug Repurposing Studies Targeting SARS-CoV-2: an Ensemble Docking Approach on Drug Target 3C-like Protease (3CL(pro)). Journal of Biomolecular Structure \&3 Dynamics 2020, 1-21. 
(20) Guo, S.; Xie, H.; Lei, Y.; Liu, B.; Zhang, L.; Xu, Y.; Zuo, Z. Discovery of Novel Inhibitors against Main Protease (Mpro) of SARS-CoV-2 via Virtual Screening and Biochemical Evaluation. Bioorganic Chemistry 2021, 110, 104767-104767.

(21) Mirza, M. U.; Froeyen, M. Structural Elucidation of SARS-CoV-2 Vital Proteins: Computational Methods Reveal Potential Drug Candidates against Main Protease, Nsp12 Polymerase and Nsp13 Helicase. Journal of Pharmaceutical Analysis 2020, 10, 320328.

(22) Delre, P.; Caporuscio, F.; Saviano, M.; Mangiatordi, G. F. Repurposing Known Drugs as Covalent and Non-covalent Inhibitors of the SARS-CoV-2 Papain-Like Protease. Frontiers in Chemistry 2020, 8, 594009-594009.

(23) Xu, X.; Liu, Y.; Weiss, S.; Arnold, E.; Sarafianos, S. G.; Ding, J. Molecular Model of SARS Coronavirus Polymerase: Implications for Biochemical Functions and Drug Design. Nucleic Acids Research 2003, 31, 7117-7130.

(24) Procacci, P.; Macchiagodena, M.; Pagliai, M.; Guarnieri, G.; Iannone, F. Interaction of Hydroxychloroquine with SARS-CoV2 Functional Proteins Using All-Atoms NonEquilibrium Alchemical Simulations. Chemical Communications 2020, 56, 8854-8856.

(25) Morse, J. S.; Lalonde, T.; Xu, S.; Liu, W. R. Learning from the Past: Possible Urgent Prevention and Treatment Options for Severe Acute Respiratory Infections Caused by 2019-nCoV. Chembiochem: a European Journal of Chemical Biology 2020, 21, 730-738.

(26) Bucci, M. Groovy RNA Polymerase. Nature Chemical Biology 2020, 16, 712.

(27) Pereira, D. A.; Williams, J. A. Origin and Evolution of High Throughput Screening. British Journal of Pharmacology 2007, 152, 53-61.

(28) Strømgaard, K., Krogsgaard-Larsen, P., Madsen, U., Eds. Textbook of Drug Design and 
Discovery, 5th ed.; CRC Press, Taylor \& Francis Group: Boca Raton; London; New York, 2017.

(29) Kuntz, I. D. Structure-Based Strategies for Drug Design and Discovery. Science 1992, 25\%, 1078-1082.

(30) Ilari, A.; Savino, C. Bioinformatics: Data, Sequence Analysis and Evolution; Humana Press: Totowa, NJ, 2008; pp 63-87.

(31) Würz, J. M.; Kazemi, S.; Schmidt, E.; Bagaria, A.; Güntert, P. NMR-Based Automated Protein Structure Determination. Archives of Biochemistry and Biophysics 2017, 628, $24-32$.

(32) Nwanochie, E.; Uversky, V. N. Structure Determination by Single-Particle CryoElectron Microscopy: Only the Sky (and Intrinsic Disorder) is the Limit. International Journal of Molecular Sciences 2019, 20, 4186.

(33) Lewis, T. E. et al. Genome3D: Exploiting Structure to Help Users Understand their Sequences. Nucleic Acids Research 2015, 43, D382-D386.

(34) Parton, D. L.; Grinaway, P. B.; Hanson, S. M.; Beauchamp, K. A.; Chodera, J. D. Ensembler: Enabling High-Throughput Molecular Simulations at the Superfamily Scale. PLoS Computational Biology 2016, 12, e1004728-e1004728.

(35) AlQuraishi, M. AlphaFold at CASP13. Bioinformatics 2019, 35, 4862-4865.

(36) Jumper, J. et al. Highly Accurate Protein Structure Prediction with AlphaFold. Nature 2021, https://doi.org/10.1038/s41586-021-03819-2.

(37) Knegtel, R.; Kuntz, I.; Oshiro, C. Molecular Docking to Ensembles of Protein Structures. Journal of Molecular Biology 1997, 266, 424-440. 
(38) Cossins, B. P.; Hosseini, A.; Guallar, V. Exploration of Protein Conformational Change with PELE and Meta-Dynamics. Journal of Chemical Theory and Computation 2012, 8, 959-965.

(39) Legina, M. S.; Nogueira, J. J.; Kandioller, W.; Jakupec, M. A.; González, L.; Keppler, B. K. Biological Evaluation of Novel Thiomaltol-Based Organometallic Complexes as Topoisomerase II $\alpha$ Inhibitors. JBIC Journal of Biological Inorganic Chemistry 2020, 25, 451-465.

(40) Wang, J.; Arantes, P. R.; Bhattarai, A.; Hsu, R. V.; Pawnikar, S.; Huang, Y. M.; Palermo, G.; Miao, Y. Gaussian Accelerated Molecular Dynamics: Principles and Applications. WIREs Computational Molecular Science 2021, e1521.

(41) Palmisano, V. F.; Gómez-Rodellar, C.; Pollak, H.; Cárdenas, G.; Corry, B.; Faraji, S.; Nogueira, J. J. Binding of Azobenzene and p-diaminoazobenzene to the Human VoltageGated Sodium Channel Nav1.4. Physical Chemistry Chemical Physics 2021, 23, 35523564 .

(42) Walters, W. P.; Stahl, M. T.; Murcko, M. A. Virtual Screening - An Overview. Drug Discovery Today 1998, 3, 160-178.

(43) Amaro, R. E.; Baudry, J.; Chodera, J.; Demir, O.; McCammon, J. A.; Miao, Y.; Smith, J. C. Ensemble Docking in Drug Discovery. Biophysical Journal 2018, 114, $2271-2278$.

(44) Kalenkiewicz, A.; Grant, B. J.; Yang, C.-Y. Enrichment of Druggable Conformations from Apo Protein Structures Using Cosolvent-Accelerated Molecular Dynamics. Biology (Basel) 2015, 4, 344-366.

(45) Teague, S. J. Implications of Protein Flexibility for Drug Discovery. Nature Reviews Drug Discovery 2003, 2, 527-541. 
(46) Carlson, H. A.; McCammon, J. A. Accommodating Protein Flexibility in Computational Drug Design. Molecular Pharmacology 2000, 57, 213-218.

(47) Koshland, D. E. Application of a Theory of Enzyme Specificity to Protein Synthesis. Proceedings of the National Academy of Sciences of the United States of America 1958, $44,98-104$.

(48) Jorgensen, W. L. Rusting of the Lock and Key Model for Protein-Ligand Binding. Science 1991, 254, 954-955.

(49) Frauenfelder, H.; Sligar, S. G.; Wolynes, P. G. The Energy Landscapes and Motions of Proteins. Science 1991, 254, 1598-1603.

(50) Tsai, C.-J.; Kumar, S.; Ma, B.; Nussinov, R. Folding Funnels, Binding Funnels, and Protein Function. Protein Science 1999, 8, 1181-1190.

(51) Uehara, S.; Tanaka, S. Cosolvent-Based Molecular Dynamics for Ensemble Docking: Practical Method for Generating Druggable Protein Conformations. Journal of Chemical Information and Modeling 2017, 5\%, 742-756.

(52) Ostrem, J. M.; Peters, U.; Sos, M. L.; Wells, J. A.; Shokat, K. M. K-Ras(G12C) Inhibitors Allosterically Control GTP Affinity and Effector Interactions. Nature (London) 2013, 503, 548-551.

(53) Oleinikovas, V.; Saladino, G.; Cossins, B. P.; Gervasio, F. L. Understanding Cryptic Pocket Formation in Protein Targets by Enhanced Sampling Simulations. Journal of the American Chemical Society 2016, 138, 14257-14263.

(54) Kuzmanic, A.; Bowman, G. R.; Juarez-Jimenez, J.; Michel, J.; Gervasio, F. L. Investigating Cryptic Binding Sites by Molecular Dynamics Simulations. Accounts of Chemical Research 2020, 53, 654-661. 
(55) Cimermancic, P.; Weinkam, P.; Rettenmaier, T. J.; Bichmann, L.; Keedy, D. A.; Woldeyes, R. A.; Schneidman-Duhovny, D.; Demerdash, O. N.; Mitchell, J. C.; Wells, J. A.; Fraser, J. S.; Sali, A. CryptoSite: Expanding the Druggable Proteome by Characterization and Prediction of Cryptic Binding Sites. Journal of Molecular Biology 2016, 428, 709-719.

(56) Trott, O.; Olson, A. J. AutoDock Vina: Improving the Speed and Accuracy of Docking with a New Scoring Function, Efficient Optimization, and Multithreading. Journal of Computational Chemistry 2010, 31, 455-461.

(57) Huang, S.; Zou, X. Ensemble Docking of Multiple Protein Structures: Considering Protein Structural Variations in Molecular Docking. Proteins, Structure, Function, and Bioinformatics 2007, 66, 399-421.

(58) Strecker, C.; Meyer, B. Plasticity of the Binding Site of Renin: Optimized Selection of Protein Structures for Ensemble Docking. Journal of Chemical Information and Modeling 2018, 58, 1121-1131.

(59) Bradley, P.; Misura, K. M. S.; Baker, D. Toward High-Resolution de Novo Structure Prediction for Small Proteins. Science 2005, 309, 1868-1871.

(60) Wang, A.; Zhang, Y.; Chu, H.; Liao, C.; Zhang, Z.; Li, G. Higher Accuracy Achieved for Protein-Ligand Binding Pose Prediction by Elastic Network Model-Based Ensemble Docking. Journal of Chemical Information and Modeling 2020, 60, 2939-2950.

(61) Bolstad, E. S. D.; Anderson, A. C. In Pursuit of Virtual Lead Optimization: The Role of the Receptor Structure and Ensembles in Accurate Docking. Proteins: Structure, Function, and Bioinformatics 2008, 73, 566-580.

(62) Shaw, D.; Dror, R.; Salmon, J.; Grossman, J.; Mackenzie, K.; Bank, J.; Young, C.; Deneroff, M.; Batson, B.; Bowers, K., et al. Millisecond-Scale Molecular Dynamics Simulations on Anton. ACM. IEEE Conference on Supercomputing (SC09). 2009. 
(63) Torrie, G.; Valleau, J. Nonphysical Sampling Distributions in Monte Carlo Free-Energy Estimation: Umbrella Sampling. Journal of Computational Physics 1977, 23, 187-199.

(64) Gullingsrud, J. R.; Braun, R.; Schulten, K. Reconstructing Potentials of Mean Force through Time Series Analysis of Steered Molecular Dynamics Simulations. Journal of Computational Physics 1999, 151, 190-211.

(65) Laio, A.; Gervasio, F. L. Metadynamics: a Method to Simulate Rare Events and Reconstruct the Free Energy in Biophysics, Chemistry and Material Science. Reports on Progress in Physics 2008, 71, 126601.

(66) Sugita, Y.; Okamoto, Y. Replica-Exchange Molecular Dynamics Method for Protein Folding. Chemical Physics Letters 1999, 314, 141-151.

(67) Schmidt, D.; Boehm, M.; McClendon, C. L.; Torella, R.; Gohlke, H. CosolventEnhanced Sampling and Unbiased Identification of Cryptic Pockets Suitable for Structure-Based Drug Design. Journal of Chemical Theory and Computation 2019, 15, 3331-3343.

(68) Arcon, J. P.; Defelipe, L. A.; Lopez, E. D.; Burastero, O.; Modenutti, C. P.; Barril, X.; Marti, M. A.; Turjanski, A. G. Cosolvent-Based Protein Pharmacophore for Ligand Enrichment in Virtual Screening. Journal of Chemical Information and Modeling 2019, 59, 3572-3583.

(69) Ghanakota, P.; Carlson, H. A. Driving Structure-Based Drug Discovery through Cosolvent Molecular Dynamics. Journal of Medicinal Chemistry 2016, 59, 10383-10399.

(70) Kimura, S. R.; Hu, H. P.; Ruvinsky, A. M.; Sherman, W.; Favia, A. D. Deciphering Cryptic Binding Sites on Proteins by Mixed-Solvent Molecular Dynamics. Journal of Chemical Information and Modeling 2017, 57, 1388-1401. 
(71) Zariquiey, F. S.; de Souza, J. V.; Bronowska, A. K. Cosolvent Analysis Toolkit (CAT): a Robust Hotspot Identification Platform for Cosolvent Simulations of Proteins to Expand the Druggable Proteome. Scientific Reports 2019, 9, 19118.

(72) Sayyed-Ahmad, A.; Gorfe, A. A. Mixed-Probe Simulation and Probe-Derived Surface Topography Map Analysis for Ligand Binding Site Identification. Journal of Chemical Theory and Computation 2017, 13, 1851-1861.

(73) D. E. Shaw Research, Molecular Dynamics Simulations Related to SARS-CoV-2. https://www.deshawresearch.com/downloads/download_trajectory_sarscov2. cgi/, 2020; D. E. Shaw Research Technical Data.

(74) Gao, Y. et al. Structure of the RNA-dependent RNA polymerase from COVID-19 virus. Science 2020, 368, 779-782.

(75) Case, D. A. et al. AMBER 18. 2018.

(76) Maier, J. A.; Martinez, C.; Kasavajhala, K.; Wickstrom, L.; Hauser, K. E.; Simmerling, C. ff14SB: Improving the Accuracy of Protein Side Chain and Backbone Parameters from ff99SB. Journal of Chemical Theory and Computation 2015, 11, 3696-3713.

(77) Jorgensen, W. L.; Chandrasekhar, J.; Madura, J. D.; Impey, R. W.; Klein, M. L. Comparison of Simple Potential Functions for Simulating Liquid Water. Journal of Chemical Physics 1983, 79, 926-935.

(78) Wang, J.; Wolf, R. M.; Caldwell, J. W.; Kollman, P. A.; Case, D. A. Development and Testing of a General Amber Force Field. Journal of Computational Chemistry 2004, 25, 1157-1174.

(79) Li, P.; Song, L. F.; Merz, K. M. Systematic Parameterization of Monovalent Ions Employing the Nonbonded Model. Journal of Chemical Theory and Computation 2015, $11,1645-1657$. 
(80) Martínez, L.; Andrade, R.; Birgin, E. G.; Martínez, J. M. PACKMOL: A Package for Building Initial Configurations for Molecular Dynamics Simulations. Journal of Computational Chemistry 2009, 30, 2157-2164.

(81) Shirts, M. R.; Klein, C.; Swails, J. M.; Yin, J.; Gilson, M. K.; Mobley, D. L.; Case, D. A.; Zhong, E. D. Lessons Learned from Comparing Molecular Dynamics Engines on the SAMPL5 Dataset. Journal of Computer-Aided Molecular Design 2017, 31, 147-161.

(82) Darden, T.; York, D.; Pedersen, L. Particle Mesh Ewald: An N. $\log (\mathrm{N})$ Method for Ewald Sums in Large Systems. Journal of Chemical Physics 1993, 98, 10089-10092.

(83) Salomon-Ferrer, R.; Götz, A.; Poole, D.; Le Grand, S.; Walker, R. Routine Microsecond Molecular Dynamics Simulations with AMBER on GPUs. 2. Explicit Solvent Particle Mesh Ewald. Journal of Chemical Theory and Computation 2013, 9, 3878-3888.

(84) Roe, D. R.; Cheatham, T. E. PTRAJ and CPPTRAJ: Software for Processing and Analysis of Molecular Dynamics Trajectory Data. Journal of Chemical Theory and Computation 2013, 9, 3084-3095.

(85) Sterling, T.; Irwin, J. J. ZINC 15 - Ligand Discovery for Everyone. Journal of Chemical Information and Modeling 2015, 55, 2324-2337.

(86) O'Boyle, N. M.; Banck, M.; James, C. A.; Morley, C.; Vandermeersch, T.; Hutchison, G. R. Open Babel: An Open Chemical Toolbox. Journal of Cheminformatics 2011, 3, 1-14.

(87) Morris, G. M.; Huey, R.; Lindstrom, W.; Sanner, M. F.; Belew, R. K.; Goodsell, D. S.; Olson, A. J. AutoDock4 and AutoDockTools4: Automated Docking with Selective Receptor Flexibility. Journal of Computational Chemistry 2009, 16, 2785-2791.

(88) Schmidtke, P.; Bidon-Chanal, A.; Luque, F. J.; Barril, X. MDpocket: Open-Source 
Cavity Detection and Characterization on Molecular Dynamics Trajectories. Bioinformatics 2011, 27, 3276-3285.

(89) Shao, J.; Tanner, S. W.; Thompson, N.; Cheatham, T. E. Clustering Molecular Dynamics Trajectories: 1. Characterizing the Performance of Different Clustering Algorithms. Journal of Chemical Theory and Computation 2007, 3, 2312-2334.

(90) Gaillard, T. Evaluation of AutoDock and AutoDock Vina on the CASF-2013 Benchmark. Journal of Chemical Information and Modeling 2018, 58, 1697-1706.

(91) Varela-Salinas, G.; García-Pérez, C. A.; Peláez, R.; Rodríguez, A. J. Visual Clustering Approach for Docking Results from Vina and AutoDock. Hybrid Artificial Intelligent Systems. Lecture Notes in Computer Science, vol 10334. Springer, Cham., 2017; pp 342-353.

(92) Rosenfeld, R. J.; Goodsell, D. S.; Musah, R. A.; Morris, G. M.; Goodin, D. B.; Olson, A. J. Automated Docking of Ligands to an Artificial Active Site: Augmenting Crystallographic Analysis with Computer Modeling. Journal of Computer-Aided Molecular Design 2003, 17, 525-536.

(93) Moret, M.; Zebende, G. Amino acid hydrophobicity and accessible surface area. Physical Review. E, Statistical, Nonlinear, and Soft Matter Physics 2007, 75, 011920-011920. 